\title{
Effects of Nose Bluntness on Hypersonic Boundary-Layer Receptivity and Stability over Cones
}

\author{
Kursat Kara* \\ Old Dominion University, Norfolk, Virginia 23529 \\ Ponnampalam Balakumar $\stackrel{\ddagger}{\ddagger}$ \\ NASA Langley Research Center, Hampton, Virginia 23581 \\ and \\ Osama A. Kandil辛 \\ Old Dominion University, Norfolk, Virginia 23529 \\ DOI: $\underline{10.2514 / 1 . J 050032}$
}

\begin{abstract}
The receptivity to freestream acoustic disturbances and the stability properties of hypersonic boundary layers are numerically investigated for boundary-layer flows over a $5^{\circ}$ straight cone at a freestream Mach number of 6.0. To compute the shock and the interaction of the shock with the instability waves, the Navier-Stokes equations in axisymmetric coordinates were solved. In the governing equations, inviscid and viscous flux vectors are discretized using a fifth-order accurate weighted-essentially-non-oscillatory scheme. A third-order accurate total-variationdiminishing Runge-Kutta scheme is employed for time integration. After the mean flow field is computed, disturbances are introduced at the upstream end of the computational domain. The appearance of instability waves near the nose region and the receptivity of the boundary layer with respect to slow mode acoustic waves are investigated. Computations confirm the stabilizing effect of nose bluntness and the role of the entropy layer in the delay of boundary-layer transition. The current solutions, compared with experimental observations and other computational results, exhibit good agreement.
\end{abstract}

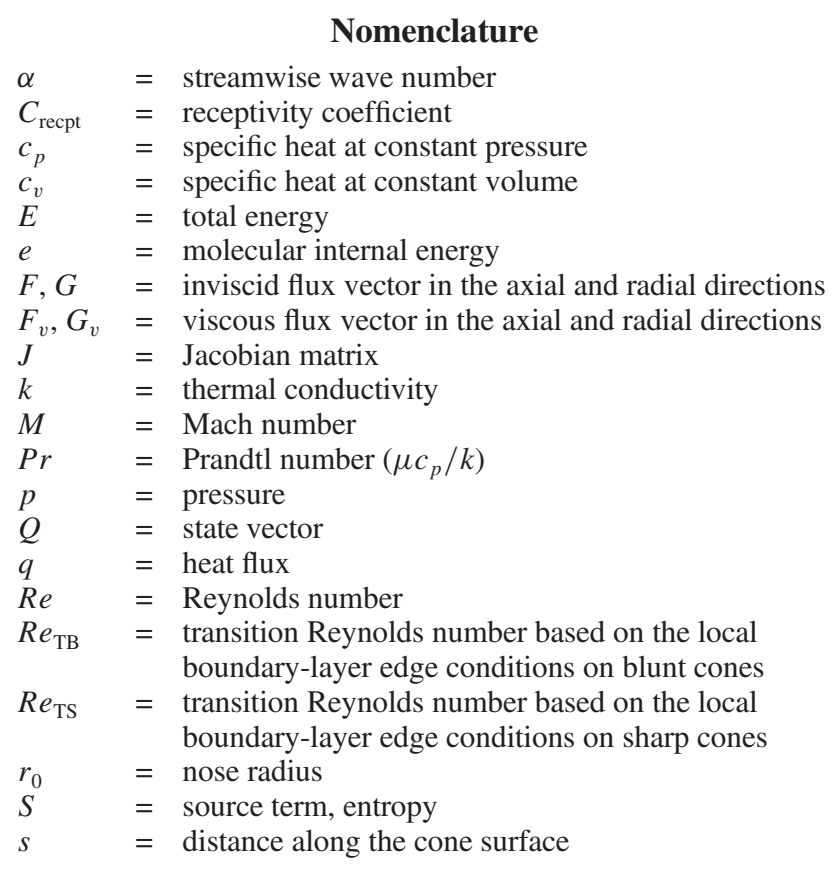

Presented as Paper 2007-4492 at the 37th AIAA Fluid Dynamics Conference and Exhibit, Miami, FL, 25-28 June 2007; received 29 June 2009; revision received 13 May 2011; accepted for publication 18 May 2011. Copyright $\odot 2011$ by Kursat Kara. Published by the American Institute of Aeronautics and Astronautics, Inc., with permission. Copies of this paper may be made for personal or internal use, on condition that the copier pay the $\$ 10.00$ per-copy fee to the Copyright Clearance Center, Inc., 222 Rosewood Drive, Danvers, MA 01923; include the code 0001-1452/11 and $\$ 10.00$ in correspondence with the CCC.

*Department of Aerospace Engineering; currently Assistant Professor, Khalifa University of Science Technology \& Research, Abu Dhabi, UAE; kursat.kara@kustar.ac.ae. Senior Member AIAA.

${ }^{\dagger}$ Research Scientist, Flow Physics and Control Branch. Member AIAA

Professor \& Eminent Scholar, Department of Aerospace Engineering. Associate Fellow AIAA

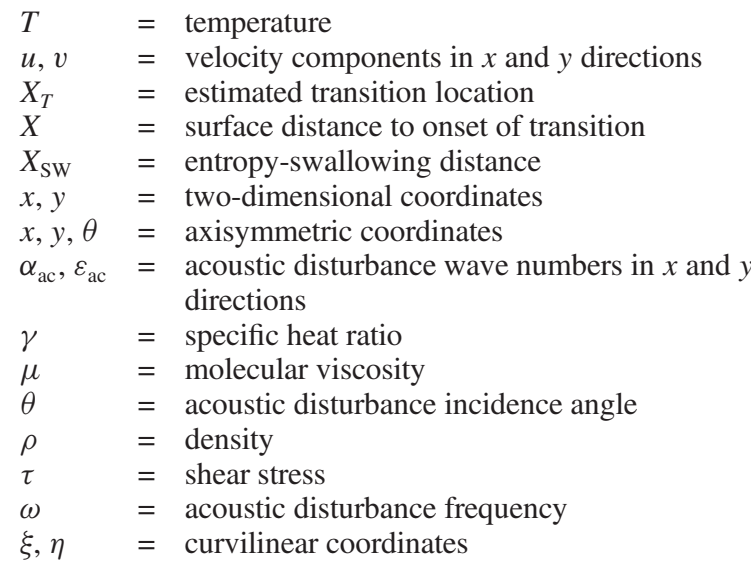

Subscripts

$\begin{array}{ll}\mathrm{ac} & =\text { acoustic } \\ e & =\text { boundary-layer edge conditions } \\ \text { recpt } & =\text { receptivity } \\ \mathrm{SW} & =\text { entropy-swallowing distance } \\ \mathrm{TB} & =\text { transition on blunt cones } \\ \mathrm{TS} & =\text { transition on sharp cones } \\ v & =\text { viscous } \\ \infty & =\text { freestream conditions }\end{array}$

\section{Introduction}

T AMINAR-TO-TURBULENT transition in shear flows occurs due to the evolution and interaction of different disturbances inside the shear layer. Though there are several mechanisms and routes to go from a laminar to a turbulent state, most of them generally follow these fundamental processes: receptivity, linear instability, nonlinear instability, saturation, secondary instability, and breakdown to turbulence.

The transition onset mainly depends on the boundary-layer characteristics and the frequency, wave number distribution, and amplitude of the disturbances that enter the boundary layer. The 
boundary-layer profiles depend on the flow parameters such as Mach number, Reynolds number, wall temperature, and model geometry. In supersonic and hypersonic boundary layers, one important geometrical parameter is nose bluntness. The effects of bluntness on transition in supersonic and hypersonic boundary layers have been studied experimentally [1-6] and numerically [7-13] by many researchers. It was identified that the entropy layer that is formed near the bow shock region persists for a long distance downstream and makes the boundary layer more stable when compared to the sharpcone case. After the entropy layer and the developing boundary layer merge together, the boundary layer becomes unstable. It was also found that, in addition to the first- and second-mode instability waves, other inviscid-type disturbances grow inside the entropy layer. It is also observed that, with increasing bluntness, the stabilizing trend is reversed in hypersonic boundary layers of cones.

Stetson [2] studied the effects of nosetip bluntness on the transition onset for sphere-cone boundary layers at freestream Mach numbers of $M=6$ and 9. The investigation mainly concentrated on the transition in the entropy-swallowing region. The variations of $X_{\mathrm{TB}} / X_{\mathrm{TS}}$ and $R e_{\mathrm{TB}} / R e_{\mathrm{TS}}$ with $X_{\mathrm{TB}} / X_{\mathrm{SW}}$ were plotted from the measurements obtained with different nose bluntness and Reynolds numbers. The results showed that, for $X_{\mathrm{TB}} / X_{\mathrm{SW}} \sim 1$, the local transition Reynolds number is about two times higher than those of a sharp cone, and the transition location is about 1.5 times higher than those of a sharp cone. For $X_{\mathrm{TB}} / X_{\mathrm{SW}} \sim 0.1$, the local transition Reynolds number is less than those of a sharp cone, and the transition location is about four to five times higher than those of a sharp cone. For $X_{\mathrm{TB}} / X_{\mathrm{SW}} \sim 0.01$, the local transition Reynolds number is very small compared to those of a sharp cone, and the transition location is about 1.5 times higher than those of a sharp cone. It is speculated that the transition in these situations is dominated by the nosetip flow and surface roughness near the nose region. Recently, Reshotko [14] attempted to explain this behavior using the transient growth theory.

Stetson et al. [3] investigated the stability of the laminar boundary layer experimentally on a $7^{\circ}$ half-angle blunt cone at a freestream Mach number of $M=8$. The experiments were performed with different nose radii of $0.15,0.25,0.50$, and $0.70 \mathrm{in}$. at a freestream unit Reynolds number of $2.5 \times 10^{6} / \mathrm{ft}$. The growth of the disturbances along the frustum were measured using hot-wire anemometry. They found that the entropy-swallowing region stabilized the boundary layer and increased the critical Reynolds number to high values. The critical Reynolds numbers increased from $5.1 \times 10^{6}$ to $10 \times 10^{6}$ when the nose radii increased from 0.15 to $0.25 \mathrm{in}$. They did not observe the transition on the body even with the smallest nose radius of 0.15 in. at this Reynolds number. They estimated that for this small bluntness case (Reynolds number based on the nose radius is 31,250), the transition Reynolds number increased by two times compared to the sharp-cone case. They also identified disturbances growing outside the boundary layer, in the entropy layer, indicating the existence of inviscid instability. They attempted to correlate this growth of the disturbances with a generalized inflection point in the entropy layer. However, the measurements did not clearly reveal any generalized inflection point in this region. This experiment was widely used in several linear stability computations [ $\underline{8}-10]$ and in direct numerical simulation (DNS) studies [11-13].

Maslov et al. [4] conducted stability experiments on sharp and blunt cones at Mach 5.92. They also measured the frequency spectra of the disturbances in the boundary layer using hot-wire anemometry. Their measurements also showed that bluntness stabilizes the second mode and delays the boundary-layer transition downstream compared to those in a sharp cone. An experimental investigation was conducted on a $5^{\circ}$ half-angle cone in a conventional Mach 6 wind tunnel by Horvath et al. [5] to examine the effects of facility noise on boundary-layer transition. In addition, the effect of bluntness at a freestream Reynolds number of $7.8 \times 10^{6} / \mathrm{ft}$ was investigated for nosetip radii $0.0001,0.03125$, and 0.0625 in. The corresponding Reynolds numbers based on nose radius are 65, 20,312, and 40,624. They found that the transition onsets shifted to $5.0,9.25$, and $12.25 \mathrm{in}$. with the increasing nose radius 0.0001 , 0.03125 , and 0.0625 in., respectively. Rufer and Schneider [6] measured mass flux profiles over $7^{\circ}$ half-angle sharp and blunt (0.020 in. radius) cones to study the amplitude and growth of instability waves. Schneider $[15,16]$ also published additional calibrated stability results of Stetson's [3] experiment.

Malik et al. [ 8 ] performed linear stability computations for Stetson's [3] experiment. They computed the mean flow by solving the parabolized Navier-Stokes equations with the initial conditions obtained from the solution of the full Navier-Stokes equations. The results were in agreement with Stetson's observations that small bluntness increases the critical Reynolds number by an order of magnitude compared to the sharp-cone value and shift the transition onset point downstream compared to the sharp-cone case. The predicted frequency of the most-amplified disturbances was about $10 \%$ higher than what was measured in the experiment. The growth rate of the most-amplified disturbances was overpredicted by about $60 \%$ compared to the experiment. It was also established by the computations that the effect of unit Reynolds number observed in the aeroballistics range data of Potter [1] was in fact a nose bluntness effect. Esfahanian [9] and Rosenboom et al. [10] also performed linear stability analysis for Stetson's experiment. Their results generally agreed with the earlier computations [] ] and overpredicted the growth rate of the most-amplified disturbances compared to those found in the experiment. Rosenboom et al. also calculated the transition onset using the $e^{N}$ method and found that the critical Reynolds number and the transition onset monotonically increased with bluntness. Based on an $N$ Factor value of 4.5 , the predicted
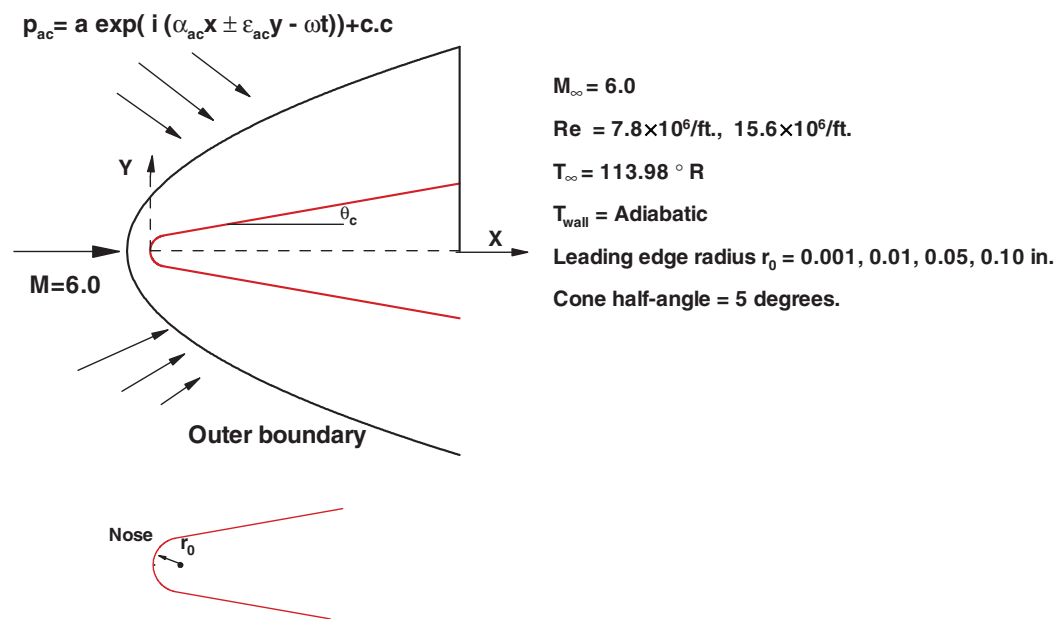

Fig. 1 Schematic diagram of the computational model. 
transition onsets for the second-mode instability shifted downstream $0.57,0.90,3.5$, and $8.8 \mathrm{~m}$ as the nose radius increased $0,3.81,17.78$, and $42.67 \mathrm{~mm}$, respectively.

Zhong and Ma [11,13] and Zhong [12] also investigated the effect of nose bluntness on the linear stability and the receptivity of the hypersonic flow over Stetson's blunt cone. They performed detailed linear stability analysis and DNS studies of wave developments inside the boundary layer. They superimposed unsteady-plane fastacoustic waves in the freestream and investigated the evolution of different modes inside the boundary layer. Their linear stability calculations were comparable to the previous computations [8-10]. Their receptivity simulations revealed that the fast acoustic modes generate the stable modes near the nose region and, as these modes evolve downstream, they generate the Mack's second mode near the synchronization point in the eigenvalue diagram.

In previous work $[17,18]$, we have investigated the interaction of forced slow and fast acoustic waves with hypersonic boundary layers over sharp-flat plates, wedges, and cones. The results showed that for flows over sharp-flat plate cases, the instability waves are generated very close to the leading edge, and the receptivity coefficient of the instability waves generated by the slow acoustic wave is about five times the amplitude of the freestream acoustic waves. It was also found that the receptivity coefficient of the slow acoustic wave is 20 times larger than the fast acoustic waves. For flows over sharp-cone cases, the receptivity coefficient is about 10 for slow acoustic waves, and for the fast acoustic waves it is about 67 times smaller. Balakumar [19] performed computations for a blunt flat plate with thicknesses of 0.0001 to $0.01 \mathrm{in}$. and a wedge of $10^{\circ}$ half-angle with different leading edge radii of 0.001 and 0.01 in. to find the effect of nose bluntness on the stability of two-dimensional supersonic boundary layers at a freestream Mach number of $M=3.5$. He found that bluntness had a strong stabilizing effect on two-dimensional boundary layers and that the boundary layers on wedges were far more stable than on blunt flat plates.

The objective of this work is to investigate the effect of nose bluntness on the hypersonic boundary layer over blunt cones and to estimate the transition Reynolds number based on the $e^{N}$ criteria, as well as to compute the receptivity coefficient of the instability waves generated inside the boundary layer. To investigate the effect of the Reynolds number based on nose radii, simulations were performed for different leading edge radii, where $r_{0}=0.001,0.01,0.05$, and $0.10 \mathrm{in}$. at a unit Reynolds number of $7.8 \times 10^{6} / \mathrm{ft}$ for a $5^{\circ}$ half-angle cone. To differentiate the unit Reynolds number effect from the nose Reynolds number effect, one simulation is performed at a higher unit Reynolds number of $15.6 \times 10^{6} / \mathrm{ft}$ with 0.05 in. nose bluntness. These parameters yield Reynolds numbers based on the nose radius that vary from 650 to 130,000 . The results consist of (1) mean flow profiles, linear stability, and transition onset Reynolds numbers for different nose radii and (2) receptivity coefficients for different nose radii.

In an earlier study by the current authors [18], our simulation code was validated against the experimental and theoretical data available in the literature. In that work, steady flow features such as shock standoff distance, bow shock shape, and wall-to-total temperature ratios were compared to experimental results and very good agreement was found. Density profiles at various downstream locations were also compared to self-similar boundary-layer solutions for each simulation and very good agreement was found.
For this study, we have selected the flow conditions and geometry used by Horvath et al. [5]. A schematic diagram of the computational setup is depicted in Fig. 1. The nose radius was varied between $r_{0}=0.001$ and $0.10 \mathrm{in}$., and the flow near the nose was resolved by using a sufficiently dense computational grid. Boundary conditions used in these simulations are described in section III.

\section{Governing Equations}

The equations solved are unsteady, compressible, axisymmetric Navier-Stokes equations in conservative form, as shown below:

$$
\frac{\partial Q}{\partial t}+\frac{\partial F}{\partial x}+\frac{\partial G}{\partial y}=\frac{\partial F_{v}}{\partial x}+\frac{\partial G_{v}}{\partial y}+S
$$

where $Q$ is the state vector, and $F$ and $G$ are the axial- and radialdirection inviscid flux vectors given by:

$$
Q=\left[\begin{array}{c}
\rho \\
\rho u \\
\rho v \\
\rho E
\end{array}\right] \quad F=\left[\begin{array}{c}
\rho u \\
\rho u^{2}+p \\
\rho u v \\
(\rho E+p) u
\end{array}\right] \quad G=\left[\begin{array}{c}
\rho v \\
\rho v u \\
\rho v^{2}+p \\
(\rho E+p) v
\end{array}\right]
$$

and $F_{v}$ and $G_{v}$ are the axial- and radial-direction viscous and heat conduction flux vectors given by:

$$
F_{v}=\left[\begin{array}{c}
0 \\
\tau_{x x} \\
\tau_{x y} \\
u \tau_{x x}+v \tau_{x y}-q_{x}
\end{array}\right] \quad G_{v}=\left[\begin{array}{c}
0 \\
\tau_{y x} \\
\tau_{y y} \\
u \tau_{y x}+v \tau_{y y}-q_{y}
\end{array}\right]
$$

The vector $S$ contains source terms resulting from axisymmetric formulation.

The source term, viscous stresses, and heat fluxes have the following form:

$$
\begin{aligned}
& S=\frac{1}{y}\left\{\left[\begin{array}{c}
0 \\
\tau_{y x} \\
\tau_{y y}-\tau_{\theta \theta} \\
u \tau_{y x}+v \tau_{y y}-q_{y}
\end{array}\right]-\left[\begin{array}{c}
\rho v \\
\rho u v \\
\rho v^{2} \\
(\rho E+p) v
\end{array}\right]\right\} \\
& \tau_{x x}=\frac{2}{3} \frac{\mu}{R e}\left(2 \frac{\partial u}{\partial x}-\frac{\partial v}{\partial y}-\frac{v}{y}\right) \quad \tau_{y y}=\frac{2}{3} \frac{\mu}{R e}\left(2 \frac{\partial v}{\partial y}-\frac{\partial u}{\partial x}-\frac{v}{y}\right) \\
& \tau_{\theta \theta}=\frac{2}{3} \frac{\mu}{R e}\left(2 \frac{v}{y}-\frac{\partial u}{\partial x}-\frac{\partial v}{\partial y}\right) \quad \tau_{x y}=\frac{\mu}{R e}\left(\frac{\partial u}{\partial y}+\frac{\partial v}{\partial x}\right) \\
& q_{x}=-\frac{\gamma}{(\gamma-1) \operatorname{PrRe}} \frac{\partial T}{\partial x} \quad q_{y}=-\frac{\gamma}{(\gamma-1) \operatorname{PrRe}} \frac{\partial T}{\partial y}
\end{aligned}
$$

Singularity exists along the symmetry axis of $y=0$. To remove the singularity we apply l'Hopital's rule to the source term and take the limit of the resulting equations as $y \rightarrow 0$, using symmetry conditions $\frac{\partial u}{\partial y}=\frac{\partial \rho}{\partial y}=\frac{\partial T}{\partial y}=\frac{\partial p}{\partial y}=0$ and $v=0$ at $y=0$. The source term along the symmetry axis $(y=0)$ becomes:

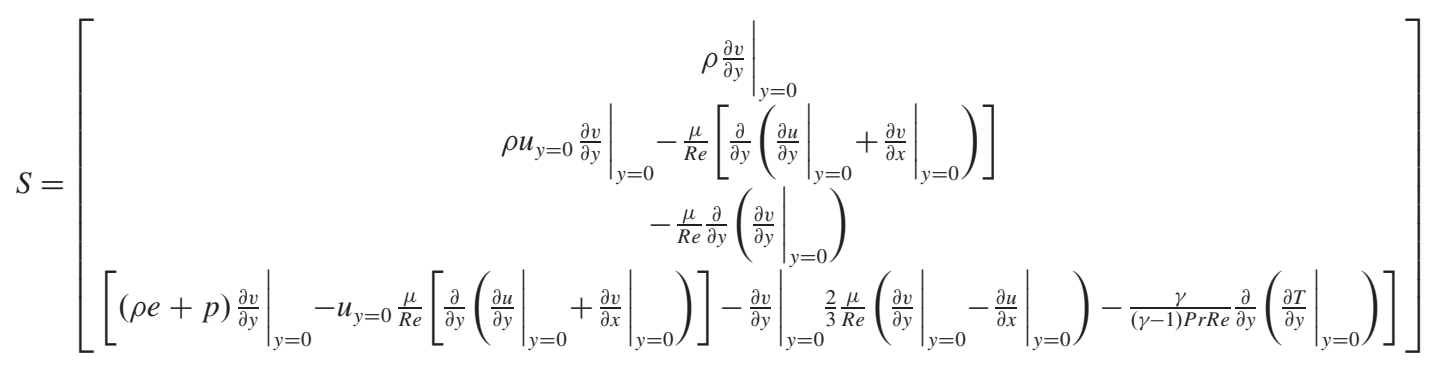


After applying the same procedure, viscous stresses and heat fluxes have the following form:

$$
\begin{aligned}
& \tau_{x x}=\frac{2}{3} \frac{\mu}{\operatorname{Re}}\left[\left.2\left(\frac{\partial u}{\partial x}-\frac{\partial v}{\partial y}\right)\right|_{y=0}\right] \\
& \tau_{y y}=\left.\frac{2}{3} \frac{\mu}{R e}\left(\frac{\partial v}{\partial y}-\frac{\partial u}{\partial x}\right)\right|_{y=0} \\
& \tau_{\theta \theta}=\left.\frac{2}{3} \frac{\mu}{R e}\left(\frac{\partial v}{\partial y}-\frac{\partial u}{\partial x}\right)\right|_{y=0} \quad \tau_{x y}=0 \\
& q_{x}=-\left.\frac{\gamma}{(\gamma-1) \operatorname{PrRe}} \frac{\partial T}{\partial x}\right|_{y=0} \quad q_{y}=0
\end{aligned}
$$

Here, $(x, y)$ represent the two-dimensional coordinates, $(x, y, \theta)$ are the axisymmetric coordinates, $(u, v)$ are the corresponding velocity components, $\rho$ is density, and $p$ is pressure. The total energy $E$ is:

$$
E=e+\frac{u^{2}+v^{2}}{2} \quad e=c_{v} T, \quad p=\rho R T
$$

Here, $e$ is the molecular internal energy and $T$ is temperature. The viscosity $(\mu)$ is computed using Sutherland's law $(\mu=$ $c_{1} T^{3 / 2} /\left(c_{2}+T\right)$, where $c_{1}=7.30246 \mathrm{E}-7$ and $\left.c_{2}=198.7^{\circ} \mathrm{R}\right)$ with no correction for low temperature, and the coefficient of conductivity is given in terms of Prandtl number $(\mathrm{Pr}=0.7)$. For computation purposes, the equations are transformed from the physical curvilinear coordinate system $(x, y)$ to a computational coordinate system $(\xi, \eta)$. The transformed governing equations are:

$$
\frac{\partial \bar{Q}}{\partial t}+\frac{\partial \bar{F}}{\partial \xi}+\frac{\partial \bar{G}}{\partial \eta}=\frac{\partial \bar{F}_{v}}{\partial \xi}+\frac{\partial \bar{G}_{v}}{\partial \eta}+\bar{S}
$$

The individual components are:

$$
\begin{gathered}
\bar{Q}=\frac{Q}{|J|}, \quad \bar{F}=\frac{J}{|J|} F, \quad \bar{G}=\frac{J}{|J|} G, \quad \bar{F}_{v}=\frac{J}{|J|} F_{v} \\
\bar{G}_{v}=\frac{J}{|J|} G_{v}, \quad \bar{S}=\frac{S}{|J|} \quad \text { and } \quad J=\left[\frac{\partial(\xi, \eta)}{\partial(x, y)}\right]
\end{gathered}
$$

\section{Solution Algorithm}

In the governing equations, inviscid and viscous fluxes were discretized using a fifth-order accurate weighted-essentially-nonoscillatory (WENO) scheme, and a third-order total-variationdiminishing (TVD) Runge-Kutta (RK) scheme was employed for time integration. Shu [20] explains the WENO and the TVD methods and the formulas. Atkins [21] gives the application of the essentiallynon-ossillatory (ENO) method to the $N$-S equations. Balakumar et al. [22] describe in detail the solution method implemented in this computation.

These methods were suitable for flows with discontinuities or high-gradient regions. These schemes solved the governing equations discretely in a uniform structured computational domain, in which flow properties are known at the grid nodes. WENO scheme approximates the spatial derivatives in a given direction to a higher order at the nodes, using the neighboring nodal values in that direction. The TVD-RK scheme integrates the resulting equations in time to acquire point values as a function of time. Since the spatial derivatives were independent of the coordinate directions, the method could easily include other dimensions. It is well known that approximating a discontinuous function by a higher-order (two or more) polynomial generally introduces oscillatory behavior near the discontinuity, and this oscillation increases with the order of the approximation. The ENO scheme and the improvement of these WENO methods were developed to keep the higher order approximations in smooth regions and to eliminate or suppress the oscillatory behavior near the discontinuities. They were achieved by systematically adopting or selecting the stencils based on the smoothness of the function, which was being approximated.

At the outflow boundary, an extrapolation boundary condition was used. No-slip, no-penetration, and adiabatic wall conditions are enforced at the wall. Density on the wall was computed from the continuity equation. In the mean flow computations, the simulation prescribed the freestream values at the outer boundary, which lay outside the bow shock. In the unsteady computations, the acoustic perturbations were superimposed to the uniform mean flow at the outer boundary. The procedure was to first compute the steady mean flow by performing unsteady computations, using a variable time step until the maximum residual reached a small value of $\sim 10^{-11}$. These computations used a Courant-Friedrichs-Lewy (CFL) number of 0.2 . The next step was to introduce unsteady disturbances at the upper boundary of the computational domain and to perform timeaccurate computations to investigate the interaction and evolution of these disturbances on downstream direction.

The two-dimensional computational grid is generated using orthogonal curvilinear coordinates $(\xi, \eta)$, representing downstream and wall-normal coordinates, respectively. The grid was stretched in the $\eta$ direction, close to the wall, and was uniform outside of the boundary layer. In the $\xi$ direction, the grid was very fine near the nose and was uniform in the flat region. For the simulation, it was assumed that the flow is axisymmetric. The outer boundary that lay outside the shock followed a parabola, so that the boundary-layer growth could be captured accurately. The computational domain extended from $x=-0.015$ to $36.0 \mathrm{in}$. in the axial direction, depending on the bluntness. Calculations were performed using a grid that had 32 blocks with $127 \times 251$ grid points for each block, where the computational domain has approximately one million grid points. Grid refinement studies were also performed with different grid distributions in the $x$ and $y$ directions. Because of the very fine grid requirement near the nose, the allowable time step was very small and the computations became too expensive to simulate the unsteady computations in the entire domain. In the nose region, about 20,000 to 50,000 time steps per cycle were required depending on the bluntness and the frequencies that were simulated. To overcome this, calculations were performed in two steps. First, the computations were done near the nose region with a very small time step. Second, the flow properties in the middle of the domain were fed as inflow conditions for the second larger domain so that the computations were carried out with a larger time step.

The acoustic field that impinged on the outer boundary was taken to be in the following form:

$$
p^{\prime}=\operatorname{Real}\left\{\tilde{p} e^{i \alpha_{\mathrm{ac}} x \pm i \varepsilon_{\mathrm{ac}} y-i \omega t}\right\}
$$

Here $\alpha_{\mathrm{ac}}$ and $\varepsilon_{\mathrm{ac}}$ were the acoustic wave numbers in the $x, y$ directions, and $\omega$ is the frequency of the acoustic disturbance. The wave number in the $y$-direction, $\varepsilon_{\text {ac }}$, determines the incident angle of the acoustic waves; we made computations for zero incident angle $\varepsilon_{\mathrm{ac}}=0.0$.

\section{Results}

Computations were performed for hypersonic flows at a freestream Mach number of 6.0 over a $5^{\circ}$ half-angle cone with blunt noses. The flow parameters are given in Table 1 . The inviscid conditions, downstream of the shock, on the surface of a sharp cone are given in Table 2 . Figure 1 shows the schematic diagram of the computational setup. The nose region of the cone was modeled as a circle of the form:

$$
\left(x-r_{0}\right)^{2}+y^{2}=r_{0}^{2}
$$

Here, $r_{0}$ is the nose radius. Simulations were performed for the nose radii of $r_{0}=0.001,0.01,0.05$ and 0.10 in. at a unit Reynolds number of $7.8 \times 10^{6} / \mathrm{ft}$. This provides $R e_{r 0}$ (Reynolds number based on nose radius $r_{0}$ ) as $650,6500,32,500$, and 65,000 . To achieve higher $R e_{r 0}$, two computations were performed at a higher unit Reynolds number of $15.6 \times 10^{6} / \mathrm{ft}$ for the nose radii of $r_{0}=0.05$ and 0.10 in.. The various cases are summarized in Table $\underline{3}$. 
Table 1 Flow parameters for Horvath et al.'s [5] wind tunnel model

\begin{tabular}{lc}
\hline \hline Freestream & $M_{\infty}=6.0$ \\
$\quad$ Mach number & $R e_{\infty}=7.8 \times 10^{6} / \mathrm{ft}$ \\
$\quad$ Denit Reynolds number & $\rho_{\infty}=7.059 \times 10^{-3} \mathrm{lbm} / \mathrm{ft}^{3}$ \\
$\quad$ Velocity & $U_{\infty}=3140.21 \mathrm{ft} / \mathrm{s}$ \\
Reservoir Pressure & $P_{0}=475 \mathrm{psi}$ \\
Reservoir Temperature & $T_{0}=475^{\circ} \mathrm{F}$ \\
Wall temperature & Adiabatic condition \\
Prandtl number & $P r=0.70$ \\
Ratio of specific heats & $\gamma=1.4$ \\
Length scale $\left(x_{0}=0.5\right.$ in.) & $\sqrt{v_{\infty} x_{0} / U_{\infty}}=7.30882 \times 10^{-5} \mathrm{ft}$ \\
The nondimensional frequency & $F=\frac{2 \pi v_{\infty} f}{U_{\infty}^{2}}, F=1.0 \times 10^{-4} \cdot f=390 \mathrm{kHz}$ \\
Local Reynolds number $\sqrt{R e_{x}}$ & $\sqrt{\frac{U_{e} s}{v_{e}}}$ \\
\hline
\end{tabular}

Table 2 Flow parameters at the edge of the boundary layer for a sharp cone

\begin{tabular}{lcc}
\hline \hline (Var. $)_{s} /(\text { Var. })_{\infty}$ & Sharp cone & Cone, $r_{0}=0.001$ in. \\
\hline Mach number & 0.931 & 0.932 \\
Pressure ratio & 1.547 & 1.560 \\
Density ratio & 1.367 & 1.372 \\
Temperature ratio & 1.133 & 1.137 \\
\hline \hline
\end{tabular}

Table 3 Parameters used in the computations

\begin{tabular}{ccr}
\hline \hline$r_{0}$, in. & $R e_{\infty}, / 1$ & \multicolumn{1}{r}{$R e_{r 0}$} \\
\hline 0.001 & $7.8 \times 10^{6}$ & 650 \\
0.01 & $7.8 \times 10^{6}$ & 6500 \\
0.050 & $7.8 \times 10^{6}$ & 32,500 \\
0.100 & $7.8 \times 10^{6}$ & 65,000 \\
0.050 & $15.6 \times 10^{6}$ & 65,000 \\
0.100 & $15.6 \times 10^{6}$ & 130,000 \\
\hline \hline
\end{tabular}

\section{A. Linear Stability: Similarity Profiles}

The linear stability results for the axisymmetric similarity boundary layers, over the $5^{\circ}$ half-angle sharp cone at a freestream Mach number of 6.0, are presented in Fig. 2. Details about the linear stability analysis can be found in the appendix of [23]. Figure 2a shows the neutral stability diagram in $\left(\sqrt{ } R e_{x}, F\right)$ and $\left(\sqrt{ } R e_{x}, \bar{\alpha}\right)$ planes for two-dimensional disturbances. The figure shows the firstand second-mode unstable regions and the variation of the wave number with the Reynolds number. Figure $2 \mathrm{~b}$ shows the $N$-Factor curves at different frequencies. Here, the variables are nondimensionalized by the variables at the edge of the boundary layer. To

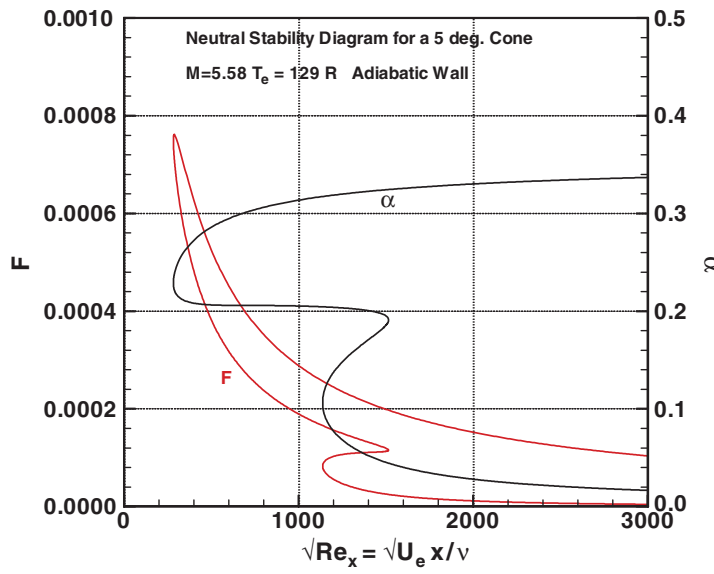

a)

Fig. 2 (a) Neutral stability diagram and variation of wave number with Reynolds number. (b) $\mathrm{N}$-Factor curves for decreasing frequency for a sharp cone. obtain the variables nondimensionalized by the freestream values as given in Table 1, the variables in this section should be multiplied by the corresponding factors from Table 2 . The frequency variable $F$ must be multiplied by 1.174 to obtain the values in terms of freestream values.

The neutral stability curve clearly shows the unstable regions at the first and second modes, and they merge at a Reynolds number of $\sqrt{ } R e_{x}=1600$ for the cone. The transition Reynolds numbers based on an $N$-Factor of 10 is about 3750 , and the most amplified frequency is $F=0.78 \times 10^{-4}$. To get the most-amplified frequency in terms of freestream values, we have to multiply $F=0.78 \times 10^{-4}$ by 1.174 , which gives $0.916 \times 10^{-4}$.

\section{B. Mean Flow}

Figure $\underline{3}$ shows the mean flow density contours. Figures $\underline{3 a}-3 \mathrm{e}$ depict the results for the $5^{\circ}$ half-angle cone at different nose radii of $r_{0}=0.001,0.05,0.10$, and 0.10 (at two times the unit Reynolds number) in. Smaller nose radius cases at $r_{0}=0.001,0.05$, and 0.10 were performed at a unit Reynolds number of $7.8 \times 10^{6} / \mathrm{ft}$, which yielded Reynolds numbers based on the nose radii varying from 650 to 65,000 . Figure $3 \mathrm{e}$ shows the results obtained at a higher unit Reynolds number of $15.6 \times 10^{6} / \mathrm{ft}$ with $r_{0}=0.10$, which yields the Reynolds number based on the nose radius of 130,000 . Figure $3 \mathrm{a}$ shows the density contours in a larger domain and the other figures show the flow field near the nose region. The boundary-layer edge values for the small bluntness case of $r_{0}=0.001$ are seen in Table 2 . The simulation results of the small bluntness case of $r_{0}=0.001$ agree very well with the results of the sharp-cone case with the conical flow assumption. One interesting observation is that the inviscid density contours and the shock locations are the same between Figs. $3 \mathrm{~d}$ and $3 \mathrm{e}$, which were obtained with the same bluntness of $r_{0}=0.10$ but at different unit Reynolds numbers of 7.8 and $15.6 \times 10^{6} / \mathrm{ft}$. This can probably be explained by the fact that freestream values were held constant so that the change in Reynolds number must have been caused by the viscosity, which does not affect the inviscid part of the flow if the boundary layers are thin. The shocks are located approximately at $0.0002,0.008$ and 0.016 in. 0.10 , respectively.

Figures $4 \mathrm{a}$ and $4 \mathrm{~b}$ show the effect of grid refinement on the density profiles for two bluntness cases $r_{0}=0.001$ and $0.10 \mathrm{in.}$ The computations were performed using 251 and 401 points in the $y$ direction and keeping the number of points in the $x$-direction the same. As can be seen, the solutions obtained from both grids are the same except near the shock, where, as expected, the shocks become sharper with the finer grid. The density profiles at different axial locations are plotted in Fig. $5 \mathrm{a}-5 \mathrm{~d}$ for different bluntness cases of $r_{0}=0.001,0.05,0.05\left(R e_{\infty} / \mathrm{ft}=15.6 \times 10^{6}\right)$, and 0.10 in. in the similarity coordinates. The compressible Blasius similarity profile is

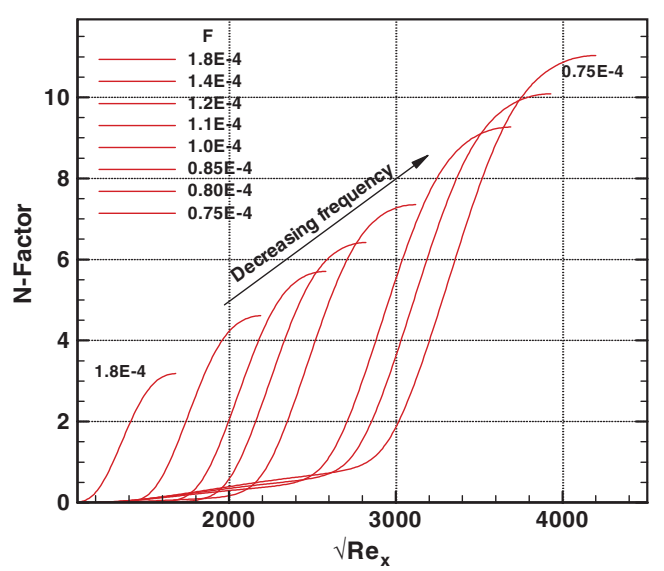

b) upstream of the leading edge for the cases of $r_{0}=0.001,0.05$, and 


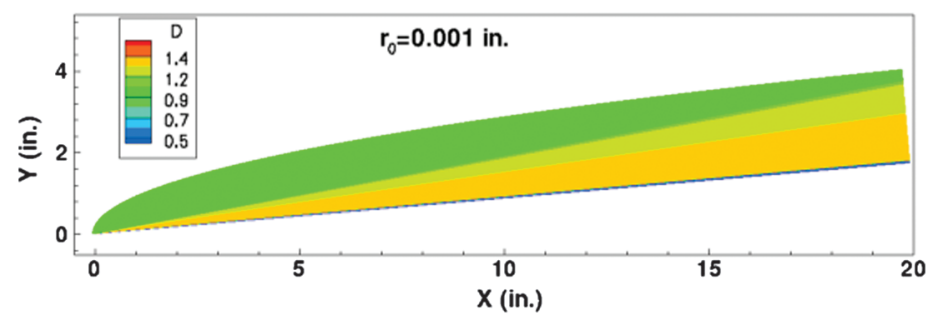

a)

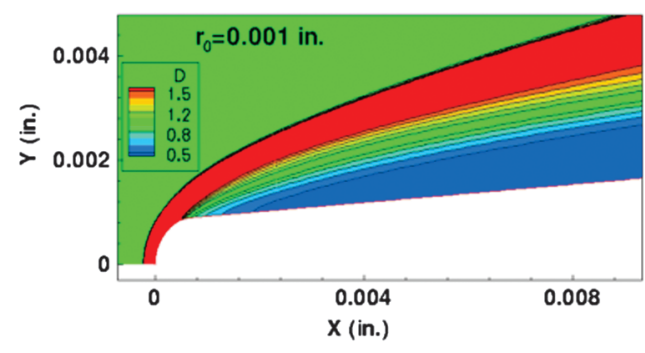

b)

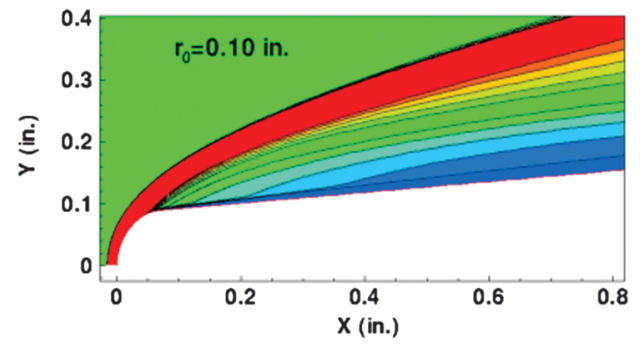

d)

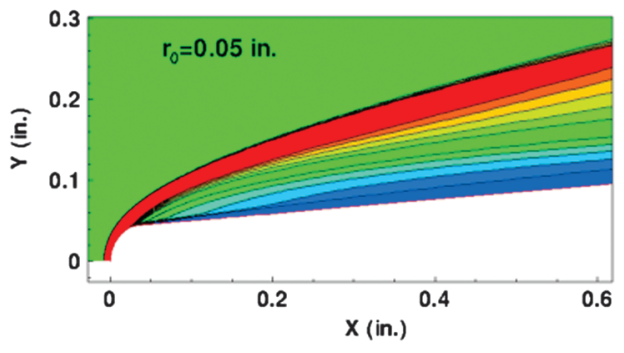

c)

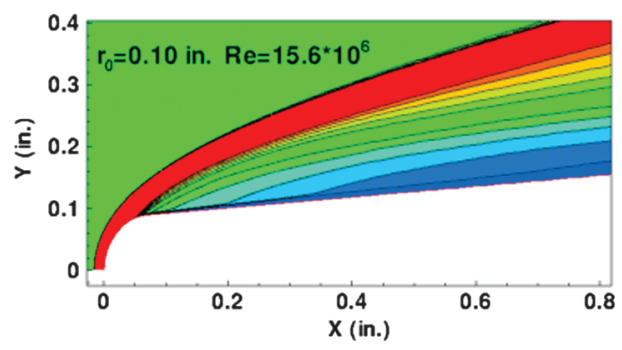

e)

Fig. 3 Contours of the density for flow over a cone with different ranges of bluntness at $M=6.0$. (a) and $(b) r_{0}=0.001$ in., $R e=7.8 \times 10^{6}$, (c) $r_{0}=0.05$ in., $R e=7.8 \times 10^{6}$, (d) $r_{0}=0.10$ in., $R e=7.8 \times 10^{6}$, (e) $r_{0}=0.10$ in., $R e=15.6 \times 10^{6}$.

also included for comparison. Here, the similarity parameter $\eta$ is defined as

$$
\eta=\frac{y_{n}}{\sqrt{\frac{v_{e} s}{U_{e}}}}
$$

where $y_{n}$ is the distance normal to the surface, $s$ is the distance along the surface of the cone, and $v_{e}$ and $U_{e}$ are the kinematic viscosity and the tangential velocity at the edge of the boundary layer. The difference between the density profiles with the bluntness and the similarity profiles increases with the bluntness. It is seen that, for the small bluntness case of $r_{0}=0.001$ in., the boundary-layer profiles approach the similarity profiles at about $x \sim 0.5 \mathrm{in}$. It was also found that, for the case of $r_{0}=0.01$ in., this occurred around $x \sim 7.0$ in. For the larger radii of 0.05 and 0.10 in., the boundary-layer profiles did not approach the similarity profiles within the respective computational domains of $x \sim 25$ and 40 in., which are closer to the transition onset points. Figures $5 \mathrm{e}$ and $5 \mathrm{f}$ depict the density profiles for $r_{0}=0.05$ and $0.10 \mathrm{in}$. in the physical coordinate.

Figures 6a-6d depict the entropy contours for different bluntness cases of $r_{0}=\overline{0.0} 01,0.05,0.10$, and $0.10\left(R e_{\infty} / \mathrm{ft}=15.6 \times 10^{6}\right)$ in. Here, the entropy is computed from

$$
S=\frac{\gamma}{\gamma-1} \ln \left(\frac{T}{T_{\infty}}\right)-\ln \left(\frac{p}{p_{\infty}}\right)
$$

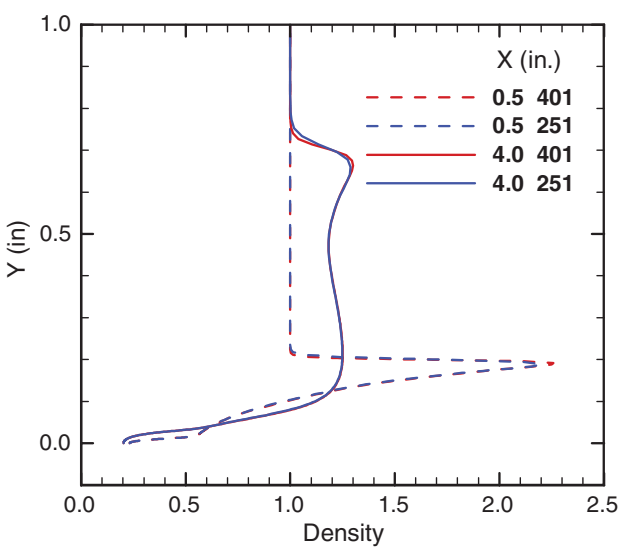

b) $r_{0}=0.10$ in.

a) $\mathbf{r}_{0}=0.001$ in.

Fig. 4 Comparison of density profiles obtained using 251 and 401 grid points in the normal direction for two different bluntness values. (a) $r_{0}=0.001$ in. and (b) $r_{0}=0.10 \mathrm{in}$. 


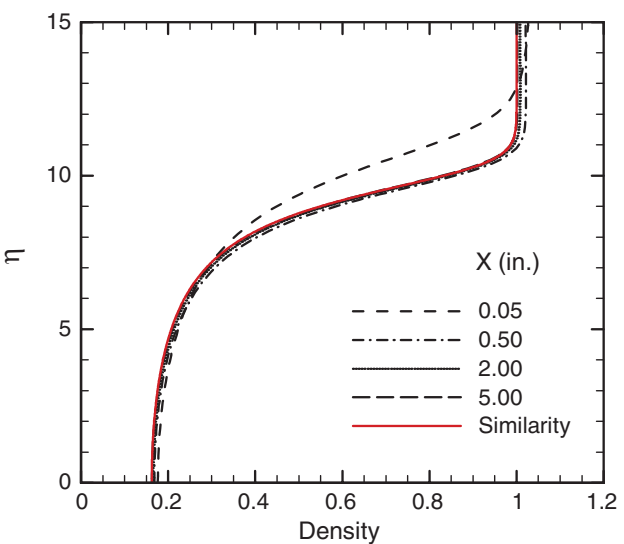

a) $r_{0}=0.001$ in.

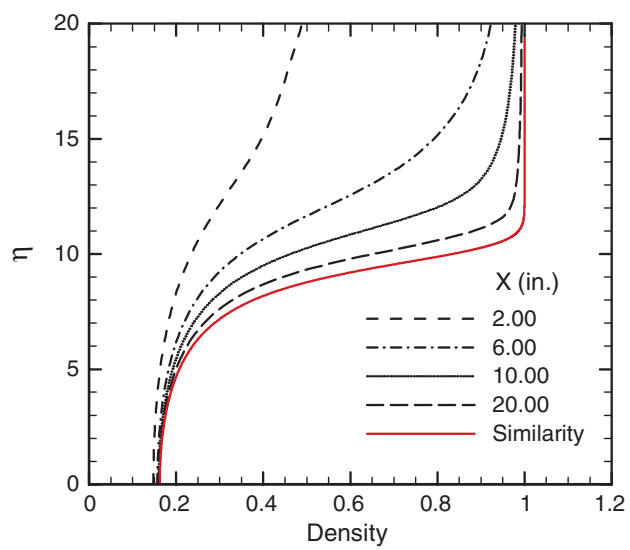

c) $r_{0}=0.05$ in. $2 \times R E$

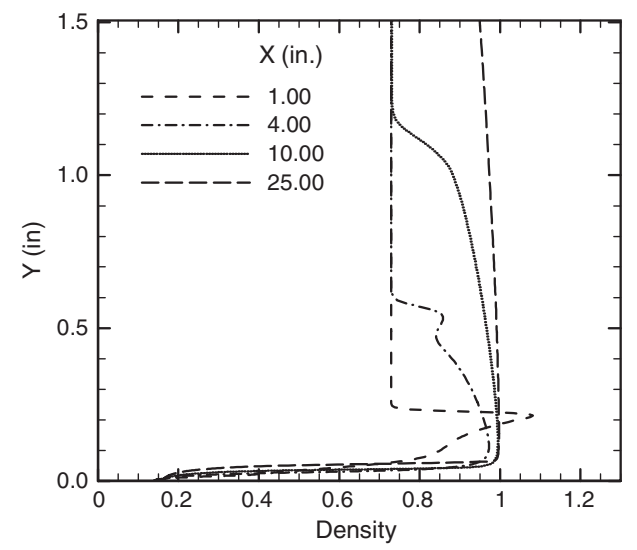

e) $r_{0}=0.05$ in.

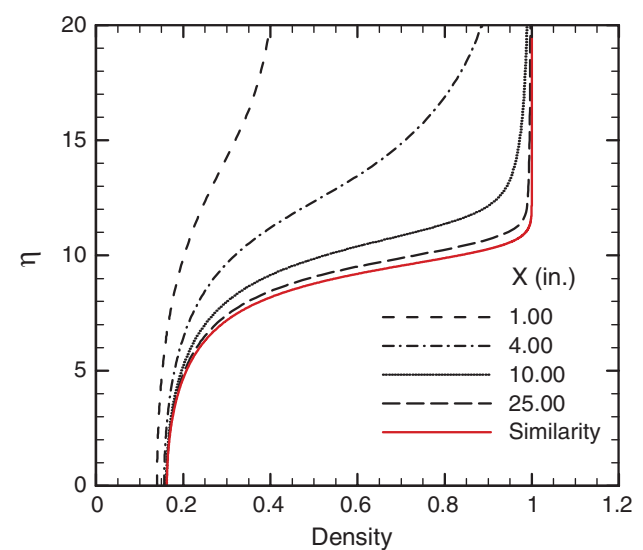

b) $r_{0}=0.05$ in.

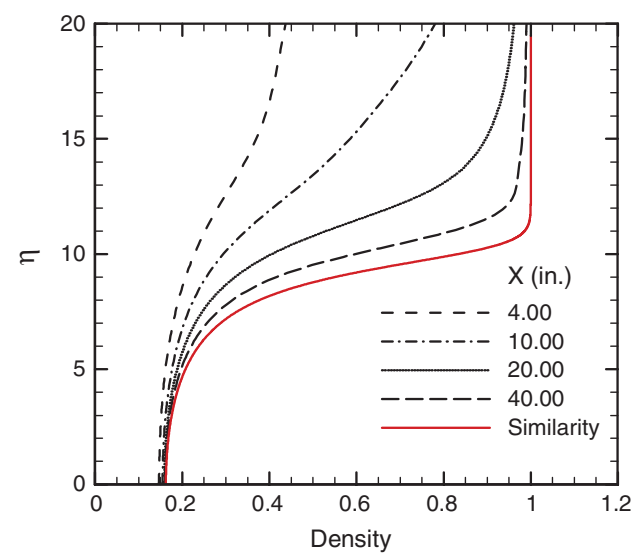

d) $r_{0}=0.10 \mathrm{in}$.

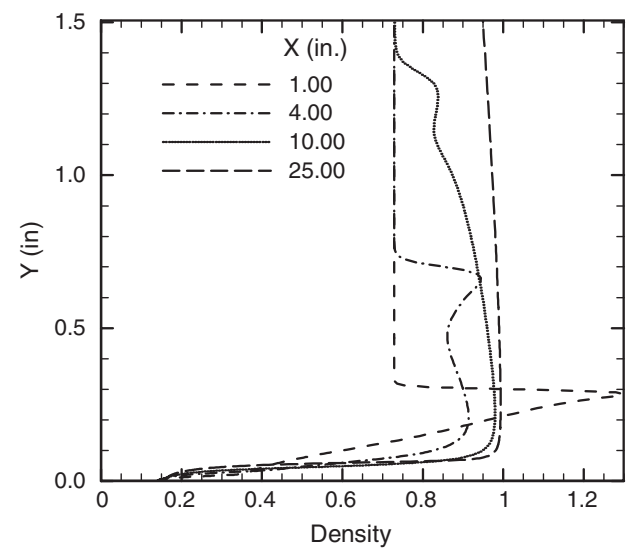

f) $r_{0}=0.10$ in.

Fig. 5 Mean density profiles at different $X$ locations using similarity coordinates for (a) $r_{0}=0.001$ in., $R e=7.8 \times 10^{6},(b) r_{0}=0.05$ in., $R e=7.8 \times 10^{6}$, (c) $r_{0}=0.05$ in., $R e=15.6 \times 10^{6}$, and (d) $r_{0}=0.10$ in., $R e=7.8 \times 10^{6}$. Density profiles versus $Y$ coordinate for (e) $r_{0}=0.05$ in., $R e=7.8 \times 10^{6}$ and (f) $r_{0}=0.10$ in., $R e=7.8 \times 10^{6}$.

For the small nose radius $r_{0}=0.001$, only the boundary layer appears near the nose region, and the entropy layer is not discernable in the outer part of the boundary layer. For the larger nose radii, the entropy layer that was visible near the nose region persisted downstream and eventually merged with the boundary layer. Figures $7 \mathrm{a}-7 \mathrm{~d}$ show the entropy profiles at different axial locations for different bluntness cases. As was observed in the contours, any discernable entropy layer also appears for the small bluntness cases. For the nose radius $r_{0}=0.05$, two layers are clearly seen in the profiles at $x=0.50$ and 1.0 in. One layer, the viscous boundary layer, exists very close to the wall with large gradients, and the other, the inviscid entropy layer produced by the bow shock near the nose, exists away from the wall with small gradients. The outside entropy layer and the boundary layer become as a single layer beyond $x=2.0$ in. As the boundary layer grows downstream, it gradually absorbs the lower part of the entropy layer, and the entropy layer shrinks and moves toward the wall. It is seen that the boundary layer completely swallowed the entropy layer by $x \sim 8$ in., and beyond that the boundary layer grows as in the sharpcone case. At larger nose radii, the two layers are more evident (marked by BL and EL in Fig. 7c), and the merging occurs at larger distances from the nose. For the bluntness case of $r_{0}=0.10$, the two layers merge close to $x=5.0 \mathrm{in}$; f for the case of $r_{0}=0.10$, with a larger unit Reynolds number, the merging occurs close to $x=5.0 \mathrm{in}$., which is equivalent to $10.0 \mathrm{in}$. when it is converted to the same unit Reynolds number. Figures $7 \mathrm{c}$ and $7 \mathrm{~d}$ show that swallowing of the boundary layers occurs at about $x \sim 20$ and 30 in., respectively, for both cases. 


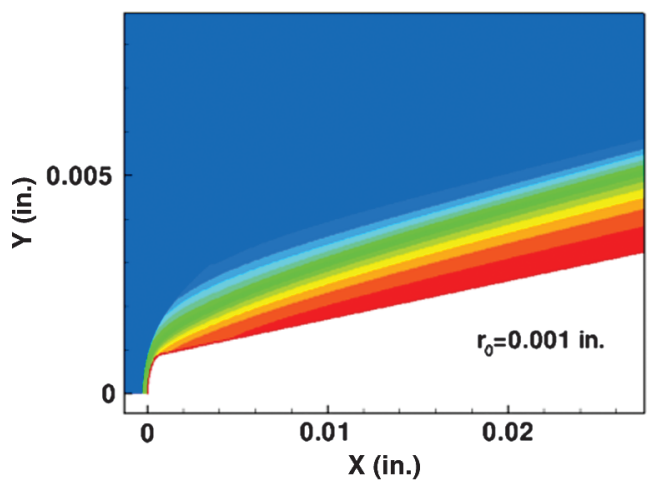

a)

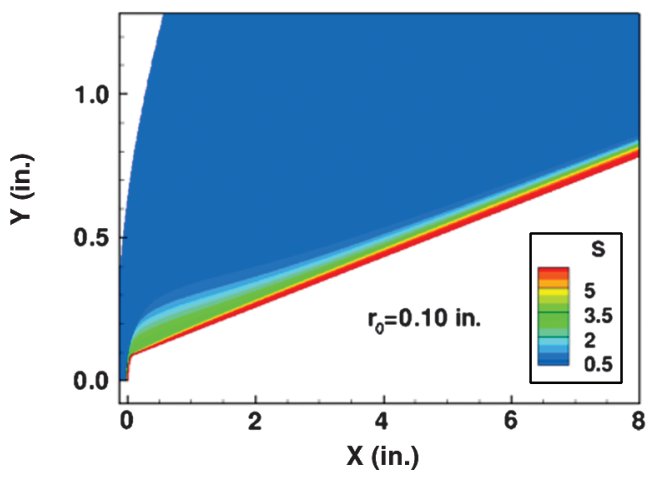

c)

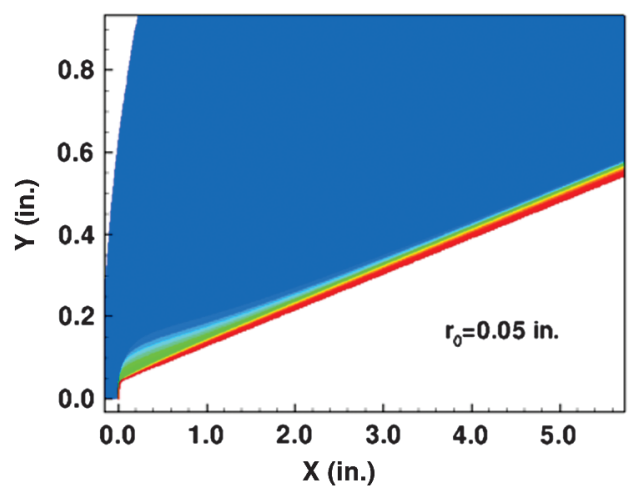

b)

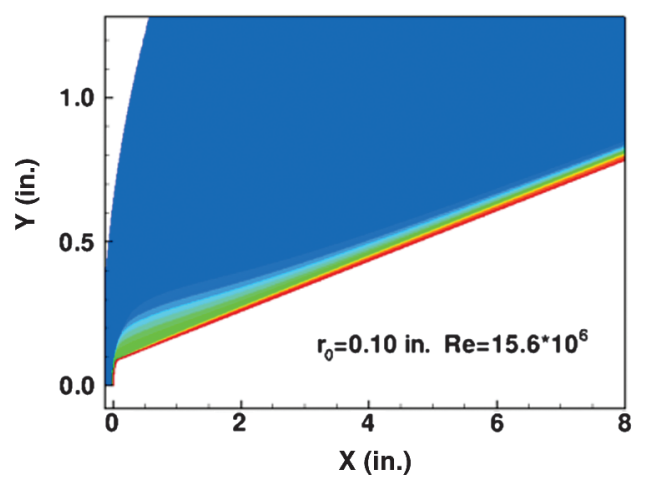

d)

Fig. 6 Contours of entropy for flow over a cone with different ranges of bluntness at $M=6.0$ (not to scale). (a) $r_{0}=0.001$ in., $R e=7.8 \times 10^{6}$, (b) $r_{0}=0.05$ in., $R e=7.8 \times 10^{6}$, (c) $r_{0}=0.10$ in., $R e=7.8 \times 10^{6}$, and (d) $r_{0}=0.10$ in., $R e=15.6 \times 10^{6}$.

\section{Linear Stability: Blunt Cones}

Figures $8 \mathrm{a}$ and $8 \mathrm{~b}$ show the $N$-Factors and the growth rates for the most-amplified disturbances [23]. They were computed using the mean profiles obtained from the numerical simulation for different

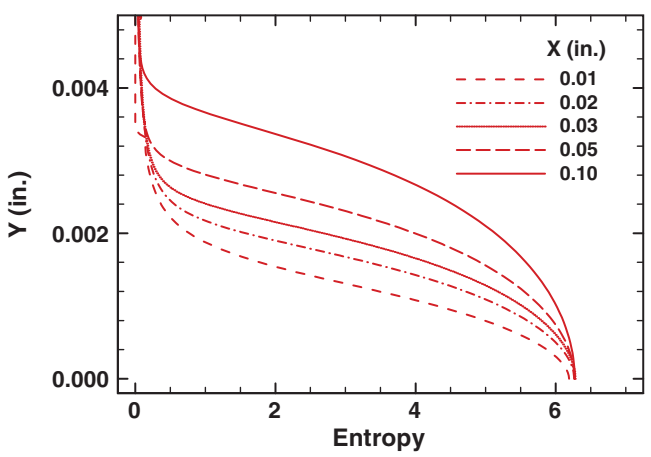

a) $r_{0}=0.001$ in.

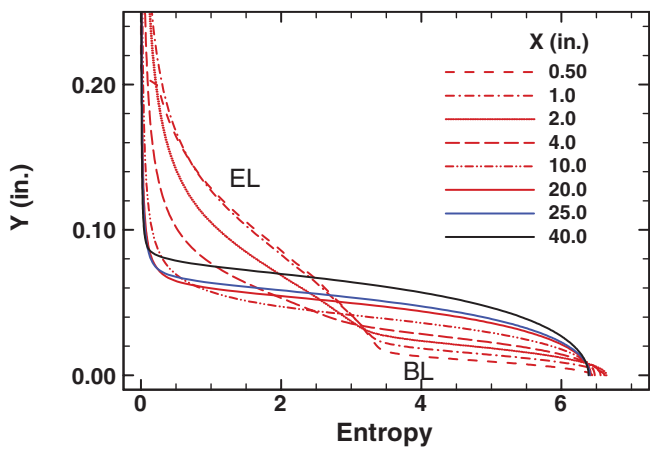

c) $r_{0}=0.10$ in. bluntness at $r_{0}=0.001,0.01,0.05$, and 0.10 with the unit Reynolds number of $7.8 \times 10^{6} / \mathrm{ft}$, as well as for $r_{0}=0.05$ and 0.10 with the higher unit Reynolds number of $15.6 \times 10^{6} / \mathrm{ft}$. For purposes of comparison, the results for the Blasius similarity profiles, which

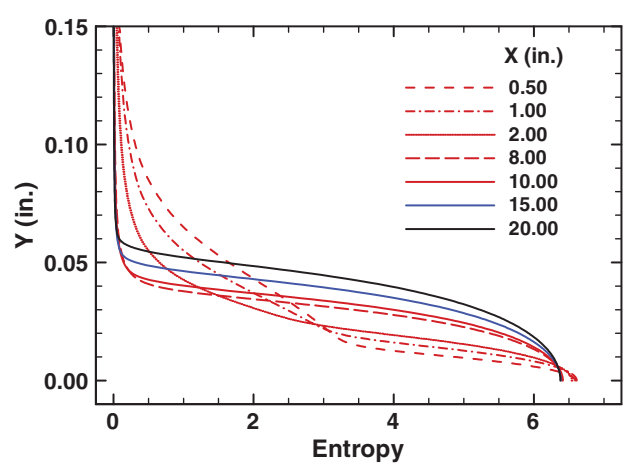

b) $r_{0}=0.05$ in.

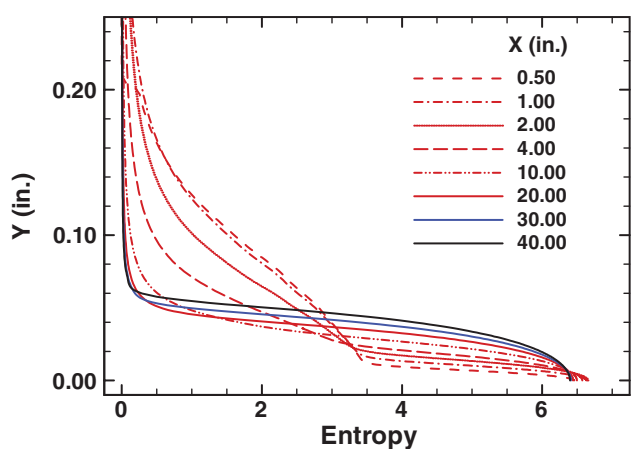

d) $\mathbf{r}_{0}=0.10$ in. $2 \times \mathbf{R E}$

Fig. 7 Entropy profiles at different $X$ stations for (a) $r_{0}=0.001$ in., $R e=7.8 \times 10^{6},(b) r_{0}=0.05$ in., $R e=7.8 \times 10^{6},(c) r_{0}=0.10$ in., $R e=7.8 \times 10^{6}$, and (d) $r_{0}=0.10$ in., $R e=15.6 \times 10^{6}$. 


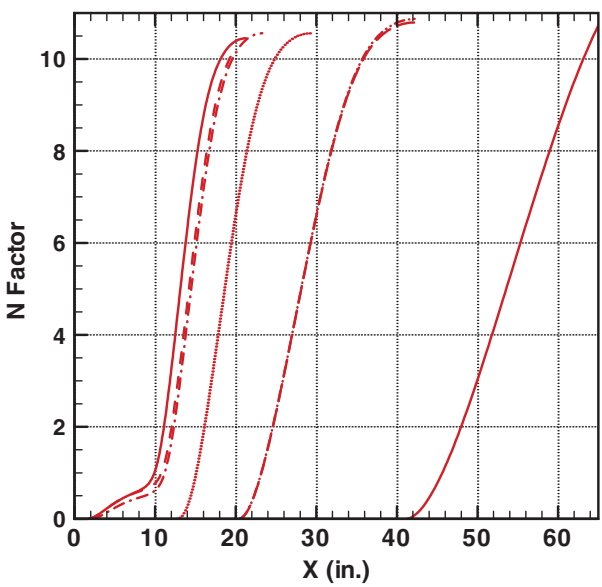

a)

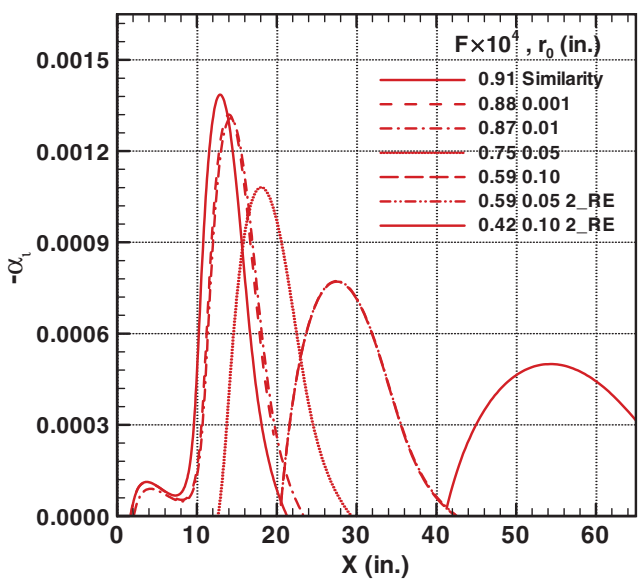

b)

Fig. 8 (a) $N$-Factors and growth rates and (b) $N$-Factors for the most-amplified disturbances for $r_{0}=0.001,0.01,0.05$, and 0.10 in. Frequencies are expressed in terms of freestream values.

Table 4 Transition Reynolds number for the blunt cones based on $N=4.5$ and 10

\begin{tabular}{lrrccccc}
\hline \hline \multirow{2}{*}{$r_{0}$, in. } & \multirow{2}{*}{$\operatorname{Re}_{r 0}$} & \multicolumn{2}{c}{$X_{T}$, in. } & \multicolumn{2}{c}{$R e_{T} \times 10^{6}$} & \multicolumn{2}{c}{$\left(R e_{T}\right)_{r o} /\left(R e_{T}\right)_{\text {Similarity }}$} \\
\cline { 3 - 8 } & & $N=4.5$ & $N=10$ & $N=4.5$ & $N=10$ & $N=4.5$ & $N=10$ \\
\hline $0.000 \mathrm{a}$ & 0 & 5.64 & 18.13 & 3.66 & 11.78 & 1.00 & 1.00 \\
$0.001 \mathrm{a}$ & 650 & 6.31 & 19.23 & 4.10 & 12.50 & 1.12 & 1.06 \\
$0.010 \mathrm{a}$ & 6500 & 6.40 & 19.60 & 4.16 & 12.74 & 1.13 & 1.08 \\
$0.050 \mathrm{a}$ & 32,500 & 12.50 & 24.78 & 8.12 & 16.11 & 2.23 & 1.37 \\
$0.100 \mathrm{a}$ & 65,000 & 23.20 & 35.80 & 15.08 & 23.27 & 4.11 & 1.97 \\
$0.100 \underline{\underline{b}}$ & 130,000 & 46.54 & 63.13 & 30.25 & 41.03 & 8.25 & 3.48 \\
$0.050 \underline{\underline{\underline{b}}}$ & 65,000 & & 35.70 & & 23.20 & & 1.97 \\
\hline \hline
\end{tabular}

${ }^{\mathrm{a}} R e_{\infty}=7.8 \times 10^{6}$

${ }^{\mathrm{b}} R e_{\infty}=15.6 \times 10^{6}$

model a sharp nose, are also shown in the figure. The frequency of the most-amplified wave is about $F=0.91 \times 10^{-4}$ for the similarity profiles. There is a significant difference both for the mean flow and the stability results with increasing bluntness. The growth rates become smaller and the $N$-Factor curves move downstream with increasing bluntness. For the smaller bluntness of $r_{0}=0.001$ and 0.01 cases, the $N$-Factor curves remain closer to the similarity curve. For the larger bluntness cases, the growth rates become smaller and the $N$-Factor curves move further to the right. The most-amplified frequencies are $0.88 \times 10^{-4}, 0.87 \times 10^{-4}, 0.75 \times 10^{-4}, 0.59 \times 10^{-4}$,

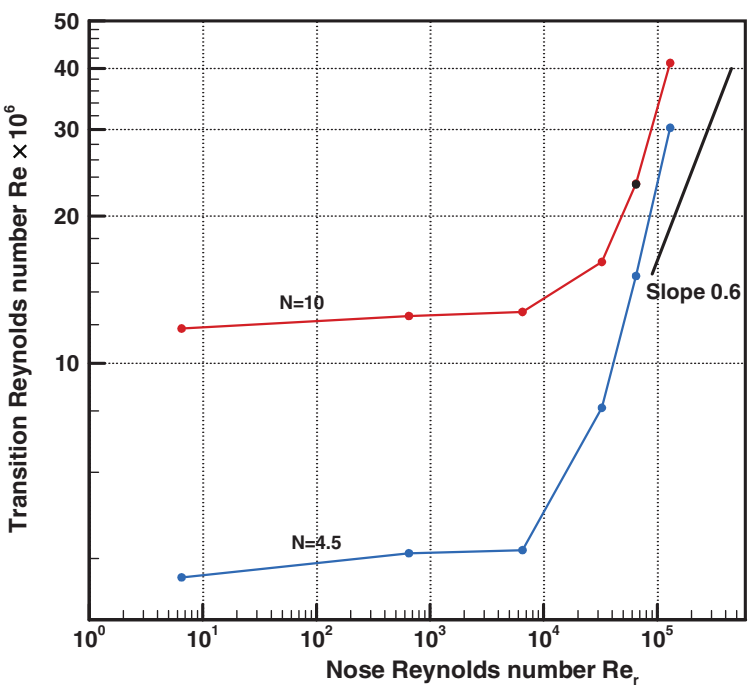

Fig. 9 The transition Reynolds numbers obtained with $N=10$ and 4.5 for different ranges of bluntness. and $0.42 \times 10^{-4}$ for $r_{0}=0.001,0.01,0.05,0.10$, and 0.10 (with higher unit Reynolds number) in., respectively. This shows that the frequencies of the most-amplified disturbances become smaller with increasing bluntness. The growth rate curves are similar to the Blasius profile for all the cases, with the exception that the mildly unstable first mode becomes stable with increasing nose bluntness. It is also seen that the window of unstable second-mode region increases with increasing bluntness. The unstable second-mode region spans from 10 to $20 \mathrm{in}$. for the sharp-cone case, and it spans from 20 to $42 \mathrm{in}$. for the blunt $r_{0}=0.10$ case. The transition Reynolds numbers obtained using the $N$-factor of 10.0 for different bluntness cases are summarized in Table 4 and plotted in Fig. 9. The ratio between the transition Reynolds number with bluntness and the transition Reynolds number for the similarity profile $\left(R e_{T}\right)_{r 0} /$ $\left(R e_{T}\right)_{\text {Similarity }}$ is $1.06,1.08,1.37,1.97$, and 3.48 , respectively, for $R e_{r 0}=650,6500,32,500,65,000$, and 130,000. To differentiate between the effects of the freestream unit Reynolds number and the nose Reynolds number, one simulation was performed for the bluntness case $r_{0}=0.05$ at twice the unit Reynolds number of $2 \times 7.8 \times 10^{6} / \mathrm{ft}$. The expectation was that the results from this simulation would be close to the results obtained for the case with $r_{0}=0.10$ at a unit Reynolds number of $7.8 \times 10^{6} / \mathrm{ft}$. It is interesting to see in Fig. 8 that the growth rates and the $N$-Factor curves are almost the same, and the most-amplified nondimensional frequencies are also the same at $F=0.59 \times 10^{-4}$. The transition Reynolds numbers are the same, $23.2 \times 10^{6}$, for the cases of $r_{0}=$ 0.10 and 0.05 (higher unit Reynolds number), respectively. The transition Reynolds number for higher unit Reynolds number case is also included as a black dot in Fig. 9. This implies that the nose Reynolds number determines the stability and the transition of boundary layers over blunt bodies. Most of the wind tunnel experiments discussed in the introduction [2-5] were performed in conventional tunnels where the freestream disturbance levels were 


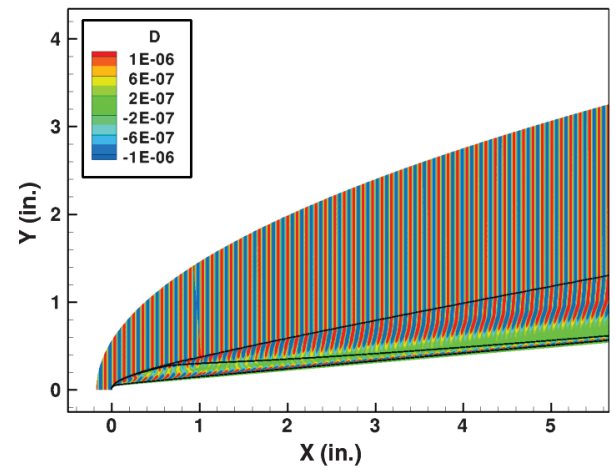

a) $\mathbf{r}_{0}=0.05$ in., $F=0.75^{*} 10^{-4}$

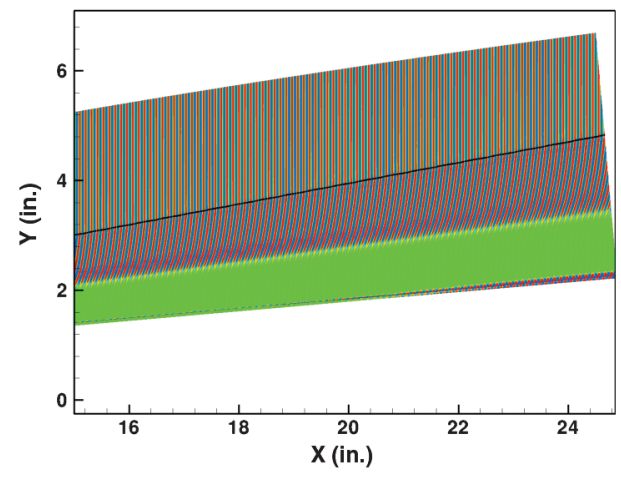

b) $\mathbf{r}_{0}=0.05$ in.

Fig. 10 Contours of the unsteady density fluctuations due to the interaction of slow acoustic wave with a blunt cone: $F=0.75 \times 10^{-4}$. (a) Nose part. (b) Flat end. Black lines represent the edge of the boundary layer, edge of the entropy layer, and the location of the shock.

high. The computed transition $N$-Factor for these tunnels varies in the range of 3.5 to $4.5[\underline{5}, \underline{8}, \underline{10}]$. Similar to the previous calculations for $N=10$, we also computed the transition onset points for different bluntness cases using $N=4.5$. The results are included in Table 4 and in Fig. 9. The ratio between the transition Reynolds number with bluntness and the transition Reynolds number for the similarity profile, $\left(R e_{T}\right)_{r 0} /\left(R e_{T}\right)_{\text {Similarity }}$, is about $1.12,1.13,2.23,4.11$, and 8.25 , respectively, for $R e_{r 0}=650,6500,32,500,65,000$, and 130,000 . The corresponding most-amplified frequencies are $1.53 \times$ $10^{-4}, 1.50 \times 10^{-4}, 0.96 \times 10^{-4}, 0.68 \times 10^{-4}$, and $0.46 \times 10^{-4}$. Horvath et al. [5] measurements showed that the transition locations shifted by $1.8 \overline{5}$ and 2.4 times the sharp-cone case as the nose
Reynolds numbers are increased to 20,312 and 40,614, respectively. Stetson's experiment [3] and the stability calculations [8] showed that the transition Reynolds number for a blunt cone at a Mach number of 8, with nose Reynolds numbers of 31,250 , increased by a factor of $1.7 \sim 2.0$ compared to a sharp cone. Rosenboom et al. [10] computations predicted, based on an $N$-Factor value of 4.5 , that transition onsets for the second-mode instability shifted downstream $1.58,6.14$, and 15.4 times the sharp-cone case as the nose Reynolds number increased to $31,250,145,800$ and 350,000 , respectively. The present predictions also fall in the same range as in the experiments and computations. Potter [1] found that, from a series of aeroballistics range experiments on nominally sharp cones, the

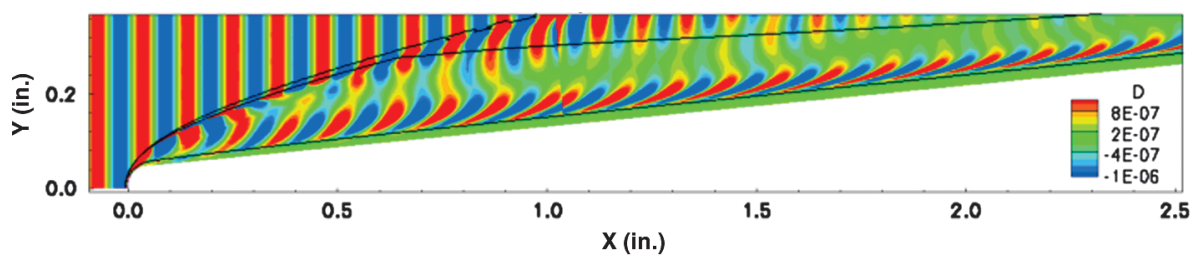

a) $\mathbf{r}_{\mathbf{0}}=\mathbf{0 . 0 5}$ in.

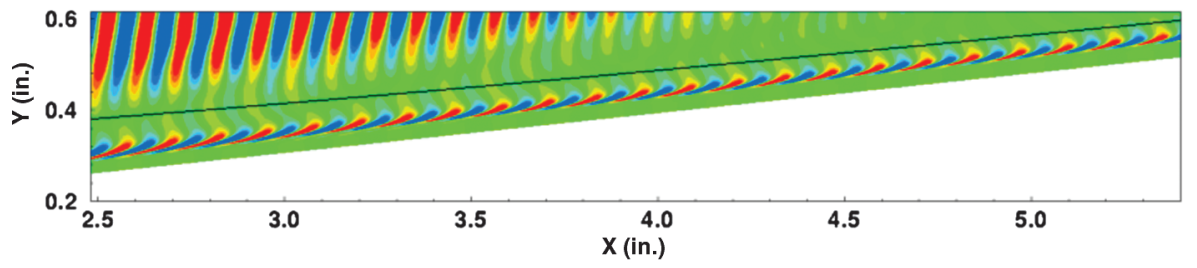

b)

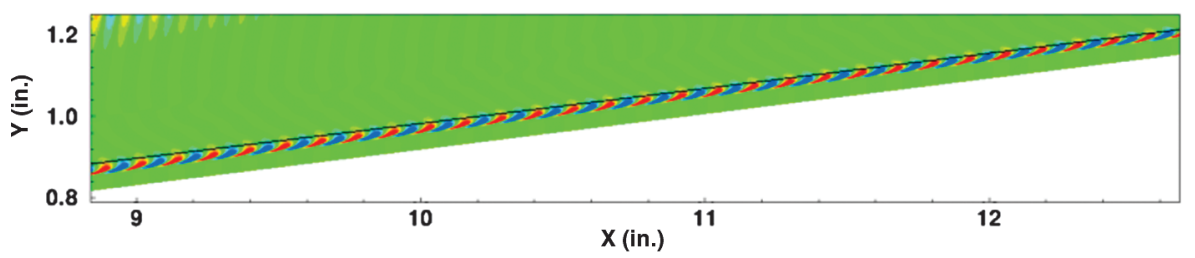

c)

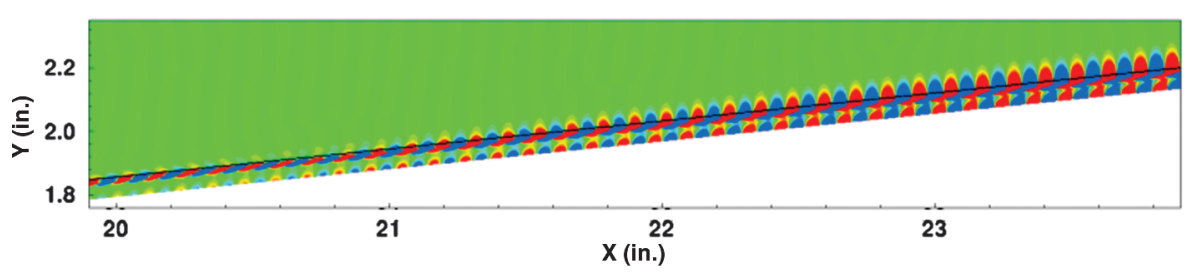

d)

Fig. 11 An expanded view of the contours with unsteady density fluctuations near the wall, along the axial direction. Black lines in (a) represent the edge of the boundary layer, edge of the entropy layer and the location of the shock; the black lines in (b), (c), and (d) represent the edge of the entropy layer. 


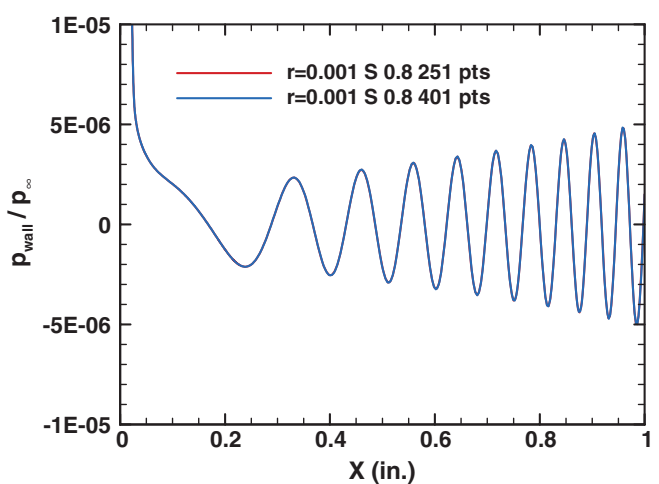

a)

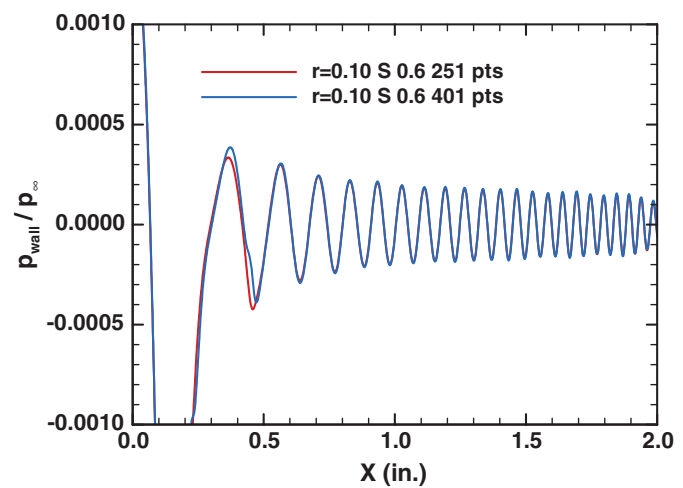

b)

Fig. 12 Comparison of unsteady pressure fluctuations along the wall, obtained using 251 and 401 grid points in the normal direction for two bluntness cases: (a) $r_{0}=0.001, F=0.80 \times 10^{-4}$ and (b) $r_{0}=0.10, F=0.60 \times 10^{-4}$.

transition Reynolds number increases with the freestream unit Reynolds number as a power of 0.63 . The effect of unit Reynolds number observed by Potter [1] may have been caused by changes in Reynolds number based on nose radius $r_{0}$. A line with the slope of 0.60 is included in Fig. 9 for comparison. The prediction from the present calculations follows this slope closely.

\section{Interaction of Slow Acoustic Waves with the Boundary Layer}

After the mean flow is computed, two-dimensional slow acoustic disturbances are introduced at the outer computational boundary and the time-accurate simulations are performed. Unsteady simulation results are first presented for the cases of $r_{0}=0.001,0.05$, $0.05\left(2 \times R e_{\infty}\right)$, and $0.10 \mathrm{in}$. at frequencies of $F=0.80 \times 10^{-4}$, $0.75 \times 10^{-4}, 0.60 \times 10^{-4}$, and $0.60 \times 10^{-4}$. These frequencies correspond to $312,292,468$, and $234 \mathrm{kHz}$, respectively, and streamwise wavelengths for slow mode correspond to 0.1007 , $0.1074,0.0671$, and 0.1343 in., respectively, for these Reynolds numbers. To remain in the linear regime, the amplitude of the forcing freestream acoustic waves was given a small value of $p_{\mathrm{ac}} / p_{\infty}=$ $2 \times 10^{-6}$. Even with this small amplitude, nonlinearity started to develop near the end of computational domain for the small bluntness case of $r_{0}=0.001$ with a frequency of $F=0.80 \times 10^{-4}$.

Figures $10 \mathrm{a}$ and $10 \mathrm{~b}$ show the results for the evolution of unsteady density fluctuations, obtained from simulations for the slow wave at a fixed time for the case $r_{0}=0.05$ and $F=0.75 \times 10^{-4}$. Figure $\underline{10 \mathrm{a}}$ shows the contours of the density fluctuations near the nose region up to $x \sim 6.0 \mathrm{in}$. and Fig. $10 \mathrm{~b}$ depicts the results near the end of the computational domain of $x=15 \sim 25$ in. Figures 11a-11d display the expanded view of the density contours near the wall along the axial direction. We also included the shock and the edge of the entropy layer in these figures. The perturbation field can be divided into four regions. One region is the area outside the shock where the acoustic waves propagate uniformly. The second region is the shock layer across which the acoustic waves are transmitted. The third region is the area between the shock and the boundary layer. This region consists of transmitted external acoustic field and the disturbances that are radiated from the boundary layer. The fourth region is the boundary layer. The figures also show that the flow field between the shock and the wall exhibit four different regions of excitations. One is the region directly below the shock where small wave diffraction occurs; the second is the region below this diffraction zone and above the entropy layer where the disturbances are quieter; the third is the entropy layer and the boundary-layer edge region near the nose where large perturbations exist; and the fourth is the region near the wall. The first important observation is that, near the nose region (Figs. 10a and 11a), the acoustic disturbances propagate across the leading edge bow shock and perturb the entropy layer. These disturbances, as they evolve downstream, remain inside the entropy layer and enter the boundary layer further downstream. Figures $11 \mathrm{~b}-11 \mathrm{~d}$ clearly show that these disturbances remain near the edge of the boundary layer for a long distance before they generate the highly unstable second mode. Another interesting observation is that the region between the boundary layer and the shock layer is quieter compared to the acoustic waves outside the shock layer. This quiet region originates from the leading-edge region (Figs. 10a and 11a), where the bow shock and the oblique shock meet. This implies that the acoustic waves are weakly transmitted through the shock and do not directly interact with the boundary layer further downstream. This was also observed in the flat plate simulation [24], where, as the acoustic wave incidence angle was increased, disturbances became quiet in the windward side.

As discussed before, grid refinement studies were performed with increasing number of points in the $y$-direction. Figures $12 \mathrm{a}$ and $12 \mathrm{~b}$ depict the effect of grid refinement on the unsteady pressure fluctuations along the wall near the nose region for two cases: $r_{0}=0.001, F=0.80 \times 10^{-4}$ and $r_{0}=0.10, F=0.60 \times 10^{-4}$. It is seen that all computations give exactly the same results and confirm that the results obtained are not due to any numerical resolutions. Figures $13 \mathrm{a}-13 \mathrm{~h}$ show the evolution of the wall pressure fluctuations for different cases. Figures 13a, 13c, 13e, and 13g show the amplitude of the pressure fluctuations along the wall in a linear scale, and Figs. 13b, 13d, 13f, and 13h depict the results in a log scale. Figures $1 \overline{\mathrm{b}}, 1 \overline{\mathrm{dd}}, 1 \overline{\mathrm{ff}}$, and $1 \overline{\mathrm{hh}}$ also include the results from the parabolized stability equations (PSE) computations obtained for the same mean boundary-layer profiles. The growth of the disturbances agrees very well with the PSE results. The figures clearly show the generation and the eventual exponential growth of the instability waves inside the boundary layer. The first observation is that there are large differences in the amplitude levels of the disturbances attained between the small bluntness case and the large bluntness cases. In all of the cases, the amplitude of the freestream acoustic pressure is the same, where $p_{\mathrm{ac}} / p_{\infty}=2 \times 10^{-6}$. For the nose radius $r_{0}=0.001$ (results shown in Figs. 13a and 13b), the disturbances grow from the leading edge and reach large amplitude levels of 0.50 near the predicted transition onset point. The slow wave, which has a wavelength that is closer to the wavelength of the instability wave, transforms into instability wave smoothly near the nose region. The parallel linear computations show that the first mode amplifies weakly up to $x \sim 10 \mathrm{in}$. for this frequency and yields an $N$-Factor of 1.0 near $x \sim 10$. However, the simulation shows that the first-mode disturbances grow much stronger near the leading edge and yield an $N$-Factor of 3.0 near $x \sim 10$. Hence, the nonparallel effects are stronger in the small bluntness case near the nose region. The maximum amplitudes obtained for the large nose radii $r_{0}=0.05$, $0.05\left(2 \times R e_{\infty}\right)$, and 0.10 (Figs. $13 \mathrm{c}-13 \mathrm{~h}$ ) are very small in the range of $\sim 10^{-4}$. The reason for this is the disappearance of the amplification of the first modes in the early part of the evolution. The disturbances not only grow but decay by two orders in magnitude before they start to grow due to the instability of second modes. This may be due to the thickening of the boundary layer. Since the fluctuations in the larger bluntness cases became very small, on the order of $10^{-8}$, we performed calculations with large freestream 


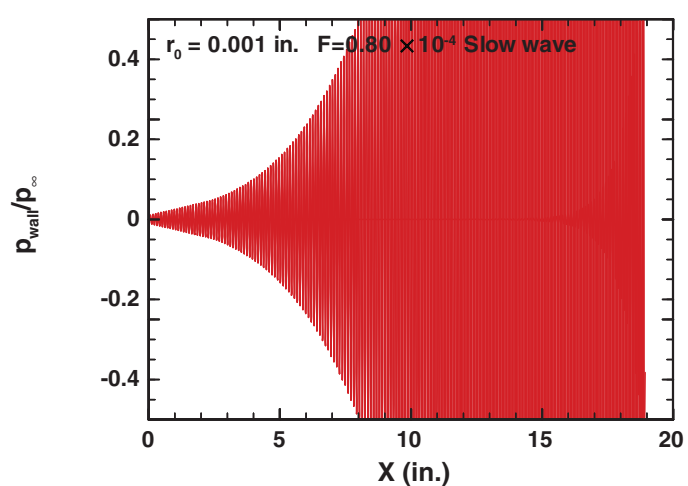

a)

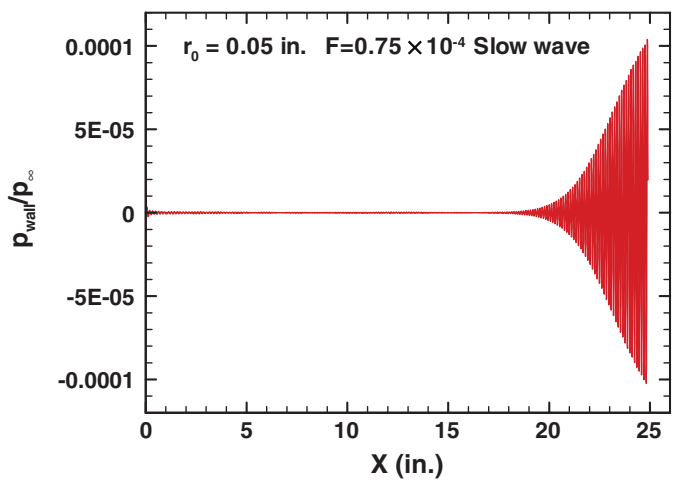

c)

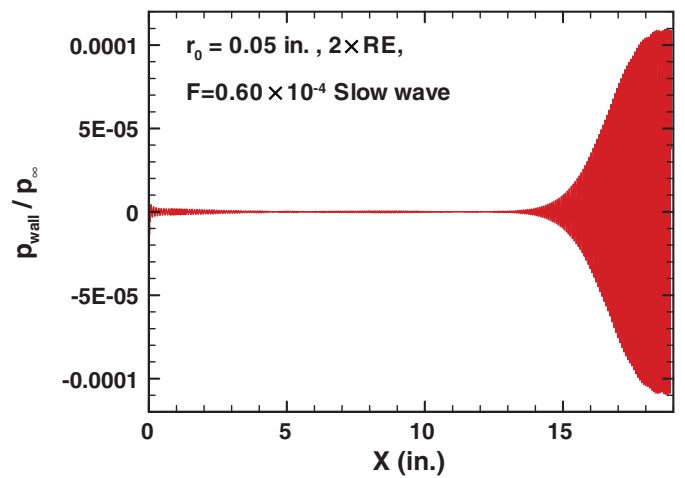

e)

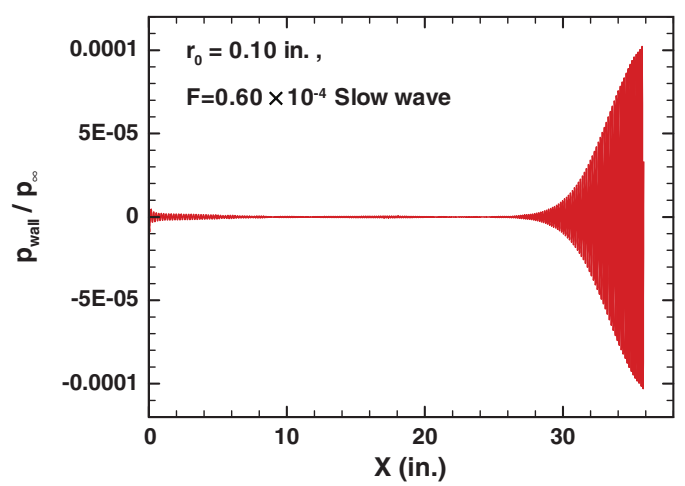

g)

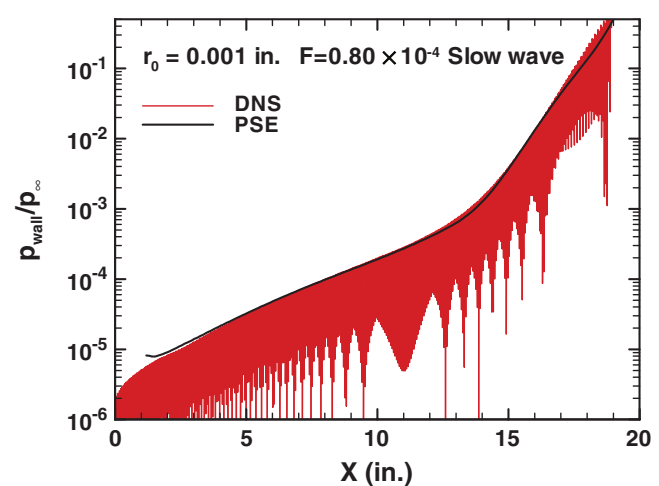

b)

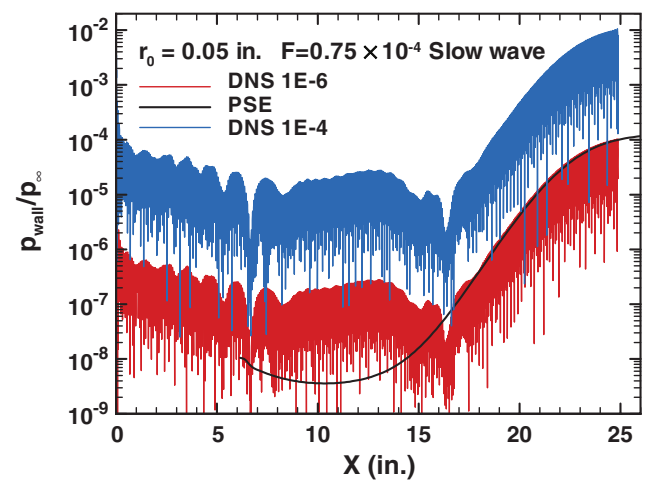

d)

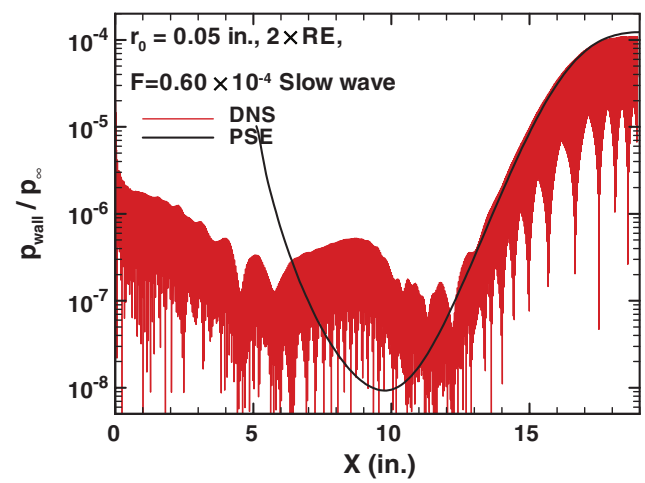

f)

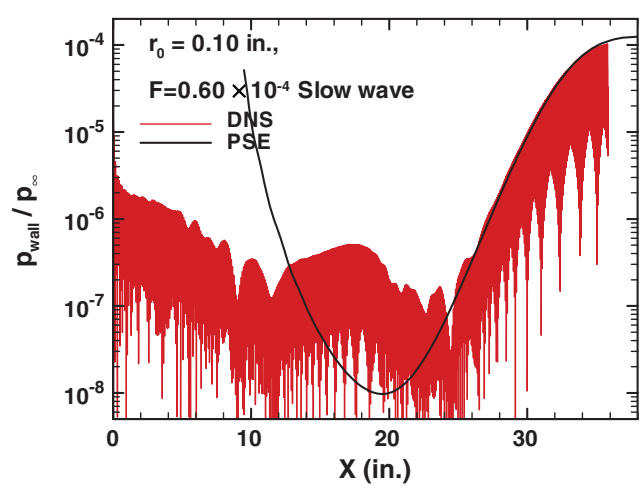

h) acoustic pressure levels at $p_{\text {ac }} / p_{\infty}=2 \times 10^{-4}$ to check the accuracy of the computations. We included the results obtained from this simulation in Fig. 13d. It should be noted that the stability properties for both freestream disturbance levels $\left(p_{\text {ac }} / p_{\infty}=2 \times 10^{-4}\right.$ and $2 \times 10^{-6}$ ) were found to be the same.

Following the PSE results up to the neutral point, the initial amplitude of the instability waves at the neutral point can be estimated. From these values, the receptivity coefficients defined by the amplitude of the pressure fluctuations at the neutral point nondimensionalized by the freestream acoustic pressure can be evaluated:

$$
C_{\text {recpt }, p_{\text {wall }}}=\frac{\left(p_{\text {wall }}\right)_{n}}{p_{\mathrm{ac}}}
$$


Table 5 Receptivity coefficients for different nose radii

\begin{tabular}{crccc}
\hline \hline$r_{0}$, in. & $R e_{r 0}$ & $F \times 10^{-4}$ & $\left(p_{\text {wall }}\right)_{n}$ & $C_{\text {recpt }, p_{\text {wall }} \text {, Slow }}$ \\
\hline $0.001^{\mathrm{a}}$ & 650 & 0.80 & $8.5 \times 10^{-6}$ & 4.23 \\
$0.050^{\mathrm{a}}$ & 32,500 & 0.75 & $3.7 \times 10^{-9}$ & $1.85 \times 10^{-3}$ \\
$0.100^{\mathrm{a}}$ & 65,000 & 0.60 & $9.5 \times 10^{-9}$ & $4.75 \times 10^{-3}$ \\
$0.050^{\mathrm{b}}$ & 65,000 & 0.60 & $10.0 \times 10^{-9}$ & $5.00 \times 10^{-3}$ \\
\hline \hline
\end{tabular}

${ }^{\mathrm{a}} R e_{\infty}=7.8 \times 10^{6}$

${ }^{\mathrm{b}} \operatorname{Re}_{\infty}=15.6 \times 10^{6}$

Table $\underline{5}$ shows the amplitude of the pressure fluctuations $\left(p_{\text {wall }}\right)_{n}$ at the neutral point and the receptivity coefficients for different nose radii. The amplitudes near the neutral points are about $8.5 \times 10^{-6}$ for the small bluntness cases and they are on the order of $10^{-9}$ for the large bluntness cases. The receptivity coefficients are 4.23, $1.85 \times 10^{-3}$, and $4.75 \times 10^{-3}$ for the cases of $r_{0}=0.001,0.05$, and 0.10 in., respectively. Another interesting observation can be made when comparing the amplitudes and the receptivity coefficients for the two cases of $r_{0}=0.10$ and $r_{0}=0.05\left(2 \times R e_{\infty}\right)$; the amplitudes and the receptivity coefficients are almost the same for these two cases. Not only for the instability behavior, but the receptivity coefficients also appear to depend only on the nose Reynolds number. This implies that unit Reynolds number effect is directly the consequence of the variation in the nose Reynolds numbers.

We have also performed additional simulations at higher frequencies for different bluntness cases. The results for the cases $r_{0}=0.001, F=1.2 \times 10^{-4}$ and $1.4 \times 10^{-4}$ were given in [18]. The conclusions are similar to the case of $F=0.80 \times 10^{-4}$ shown in Figs. $13 \mathrm{a}$ and $13 \mathrm{~b}$. The receptivity coefficients of the instability waves generated by the slow acoustic waves estimated near $x \sim$
1.0 in. are about 4.0 in both cases. Figures $14 \mathrm{a}$ and $14 \mathrm{~b}$ depict the pressure fluctuations along the wall generated by the slow acoustic waves for the cases $r_{0}=0.05, F=0.96 \times 10^{-4}$ and $r_{0}=0.10$, $F=0.68 \times 10^{-4}$, respectively. The freestream acoustic pressure amplitude is $p_{\text {ac }} / p_{\infty}=2 \times 10^{-4}$ in both cases. As mentioned previously, these frequencies correspond to the disturbances that yield the $N$-Factor of 4.5 at the earliest. Again, it is seen that the amplitudes attained by the second-mode instability waves near the end of the computational domains are on the order of $1.0 \times 10^{-4}$, which are even smaller than the freestream acoustic pressure levels.

Figure 15 shows the density fluctuations inside the boundary layer near the nose region for $r_{0}=0.001$ and $r_{0}=0.10$ (scales of different magnitude). We also included the shock and the edge of the entropy layer in these figures for illustration. The figures clearly distinguish the effect of bluntness in the generation of disturbances near the nose region. As was discussed previously, in the small bluntness case, there was no entropy layer and the disturbances excited the boundary layer up to the wall. In the large bluntness case, the disturbances are seen only away from the wall inside the entropy region.

\section{Discussion and Conclusion}

The receptivity and the stability of hypersonic boundary layers over a blunt cone with $5^{\circ}$ half-angle are numerically investigated at a freestream Mach number of 6.0 and at a Reynolds number of $7.8 \times 10^{6} / \mathrm{ft}$. Both steady and unsteady solutions are obtained by solving compressible Navier-Stokes equations using the fifth-order accurate WENO scheme for space discretization and by using a thirdorder TVD Runge-Kutta scheme for time integration. Computations are performed for different nose radii of $r_{0}=0.001,0.01,0.05,0.05$ $\left(2 \times R e_{\infty}\right), 0.10$, and $0.10\left(2 \times R e_{\infty}\right)$ in., which yields nose

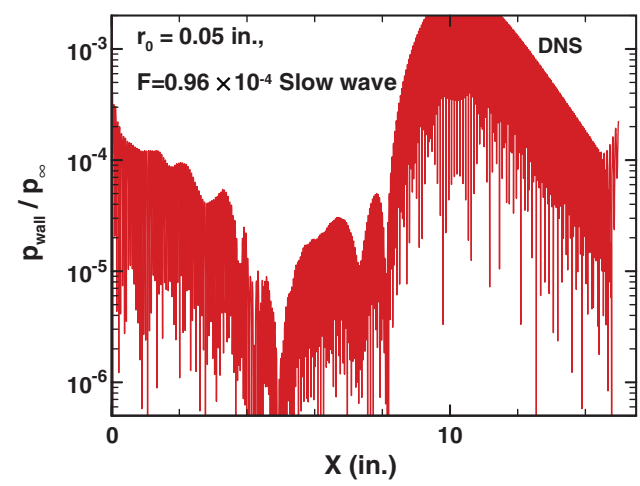

a)

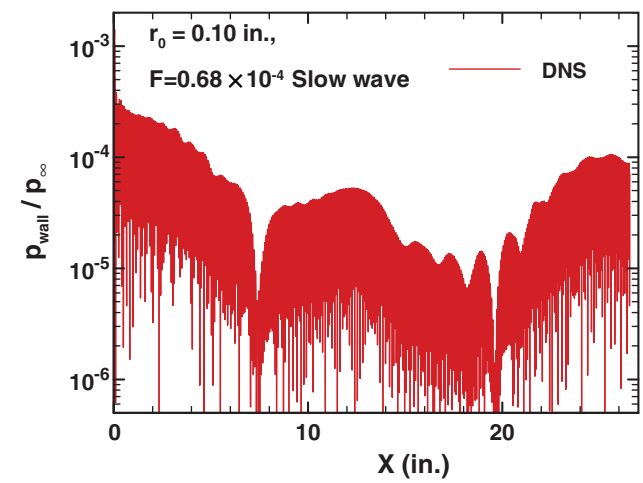

b)

Fig. 14 The amplitude of the pressure fluctuations on the wall in log-scale for the cases (a) $r_{0}=0.05, F=0.96 \times 10^{-4}$, and $(b) r_{0}=0.10$, $F=0.68 \times 10^{-4}$.

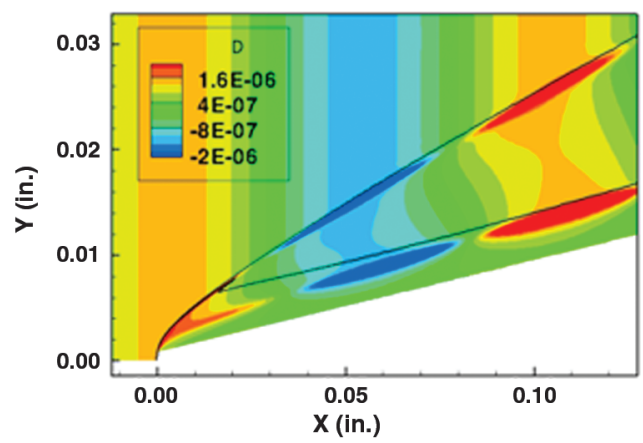

a) $r_{0}=0.001$ in., $F=0.80 * 10^{-4}$

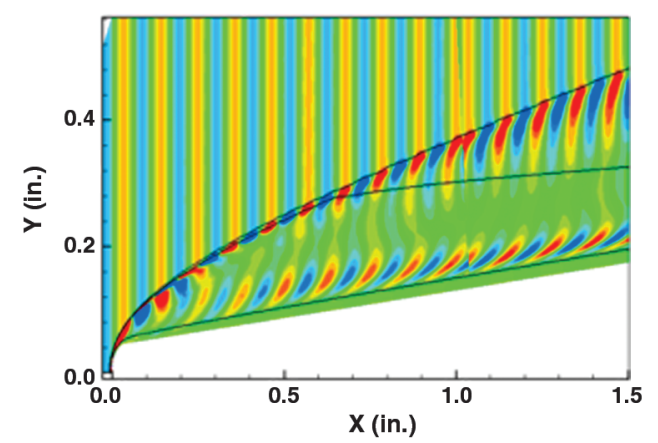

b) $r_{0}=0.10$ in., $F=0.60 * 10^{-4}$

Fig. 15 Contours of unsteady density fluctuations inside the boundary layer, near the nose region for two different bluntness cases: (a) $r_{0}=0.001$, $F=0.80 \times 10^{-4}$ and (b) $r_{0}=0.10, F=0.60 \times 10^{-4}$. Black lines represent the edge of the boundary layer, edge of the entropy layer, and the location of the shock. 
Reynolds numbers of $650,6500,32,500,65,000,65,000$, and 130,000 , respectively.

The entropy layer that forms near the nose persists over longer downstream distances with increasing bluntness and modifies the development of the boundary layer. The linear stability results show that the bluntness has a strong stabilizing effect on the stability of the boundary layers. The neutral stability points move downstream and the frequencies of the most-amplified disturbances decrease with increasing bluntness. The transition onset Reynolds number predicted using the $N$-Factor computations increases slowly up to a nose Reynolds number of 20,000 and then increases sharply at higher nose Reynolds numbers. The predicted transition Reynolds numbers using $N$ factor values of 10.0 and 4.5 are about 4.0 and 2.0 times larger than that for the Blasius boundary layer for a cone at a nose Reynolds number of 65,000 .

Unsteady simulations revealed that in the small bluntness case, amplitudes of the disturbances grow starting from the nose region and reach very large values, $\sim 0.50$, near the transition point. The computed disturbance growth agrees very well with the PSE results up to the nose region. This agreement is also further evidence that the boundary-layer disturbances in small bluntness cases originate from a small region near the nosetip since the PSE calculations were performed with a disturbance input at a single axial location. The strong growth of the first mode in the nose region leads to higher initial amplitude for the second-mode disturbance. The receptivity coefficient at a small bluntness of $r_{0}=0.001$ in. case is about 4.2. This large initial amplitude and the strong second-mode instability produce large disturbance amplitudes as the disturbances evolve in the downstream direction.

In the large bluntness cases, the important observation is that the disturbances initially decay by two to three orders of magnitude before they start to grow again due to the second-mode instability. The receptivity coefficients in large bluntness cases are much smaller in the order of $\sim 10^{-3}$. This very small initial amplitude and the weak second-mode instability produce very low disturbance amplitudes as the disturbances evolve in the downstream direction. The maximum wall pressure amplitude levels are about 50 times the freestream acoustic pressure level for the low frequency disturbances that yield $N$-Factors of 10 near the peak amplitude level locations according to linear stability theory. This factor is about 1.0 for disturbances that give $N$-Factors of 4.5. Zhong and $\mathrm{Ma}$ [13] investigated the receptivity to freestream fast acoustic waves for the flow over Stetson's [3] cone. The simulation results showed that weak second-mode waves are excited downstream of the neutral stability points. It was concluded that the second modes are generated due to the resonant interaction between the stable boundary-layer stability mode and the unstable second mode near the synchronization location between these modes. The maximum wall pressure perturbations attained by the second-mode waves in the simulations are about four times the freestream acoustic pressure levels. Wavenumber and eigenfunction analyses are not included in this paper, as was done by Zhong and Ma [13]. However, the amplitude levels they obtained agree with the present results in that the initial amplitudes of the second-mode waves are very small in hypersonic flows over blunt cones compared to sharp cones. The major conclusion from these simulations is that the mechanism considered in this investigation (the freestream planar acoustic waves will generate strong second-mode waves that will grow and cause the transition in hypersonic flows) is not supported by the findings of this study for the flows over blunt bodies. As discussed in the introduction, Horvath et al. [5] measurements showed that the transition occurred at 5.0,9.25, and 12.25 in. with the increasing nose Reynolds number of $65,20,312$ and 40,624, respectively. The question is what mechanism causes large disturbances inside the boundary layers in hypersonic flows over blunt bodies to cause the flow to become turbulent? Other types of disturbances, including oblique acoustic disturbances, three-dimensional disturbances, and roughness, may have to be considered to answer this question.

\section{Acknowledgement}

K. Kara and O. A. Kandil are supported by Flow Physics and Control Branch of NASA Langley Research Center.

\section{References}

[1] Potter, J. L., "Boundary Layer Transition on Supersonic Cones in an Aerobalistic Range," AIAA Journal, Vol. 13, No. 3, 1975, pp. 270-277. doi: $10.2514 / 3.49692$

[2] Stetson, K. F., "Nosetip Bluntness Effects on Cone Frustum Boundary Layer Transition in Hypersonic Flow," AIAA Paper 1763, 1983.

[3] Stetson, K. F., Thompson, E. R., Donaldson, J. C., and Siler, L. G., "Laminar Boundary Layer Stability Experiments on a Cone at Mach 8, Part 2: Blunt Cone," AIAA Paper 0006, 1984.

[4] Maslov, A. A., Mirinov, S. G., Shiplyuk, A. A., Sidorenko, A. A., Buntin, D. A., and Aniskin, V. M., "Hypersonic Flow Stability Experiments," AIAA Paper 0153, 2002.

[5] Horvath, T. J., Berry, S. C., Hollis, B. R., Chang, C. C., and Singer, B. A., "Boundary Layer Transition on Slender Cones in Conventional and Low Disturbance Mach 6 Wind Tunnels," AIAA Paper 2743, 2002.

[6] Rufer, S. J., and Schneider, S. P., "Hot-Wire Measurements of Instability Waves on Cones at Mach 6," AIAA Paper 3054, 2006.

[7] Reshotko, E., and Khan, M. M. S., "Stability of the Laminar Boundary Layer on a Blunted Plate in Supersonic Flow," IUTAM LaminarTurbulent Transition Symposium, Springer, Berlin, September 1979, pp. 181-200.

[8] Malik, M. R., Spall, R. E., and Chang, C. L., "Effect of Nose Bluntness on Boundary Layer Stability and Transition," AIAA Paper 0112, 1990.

[9] Esfahanian, V., "Computation and Stability Analysis of Laminar Flow over a Blunt Cone in Hypersonic Flow," Ph.D. Dissertation, The Ohio State University, Columbus, OH, 1991.

[10] Rosenboom, I., Hein, S., and Dallmann, U., "Influence of Nose Bluntness on Boundary Layer Instabilities in Hypersonic Cone Flows," AIAA Paper 3591, 1999.

[11] Zhong, X., and Ma, Y., "Receptivity and Linear Stability of Stetson's Mach 8 Blunt Cone Stability Experiments," AIAA Paper 2849, 2002.

[12] Zhong, X., "Effect of Nose Bluntness on Hypersonic Boundary Layer Receptivity over a Blunt Cone," AIAA Paper 5022, 2005.

[13] Zhong, X., and Ma, Y., "Boundary-Layer Receptivity of Mach 7.99 Flow over a Blunt Cone to Free-Stream Acoustic Waves," Journal of Fluid Mechanics, Vol. 556, No. 1, May 2006, pp. 55, 103. doi:10.1017/S0022112006009293.

[14] Reshotko, E., "Transition Issues at Hypersonic Speeds," AIAA Paper 0707, 2006.

[15] Schneider, S. P., "Hypersonic Laminar Instability on Round Cones Near Zero Angle of Attack," AIAA Paper 0206, 2001.

[16] Schneider, S. P., "Hypersonic Laminar-Turbulent Transition on Circular Cones and Scramjet Forebodies," Progress in Aerospace Sciences, Vol. 40, No. 1, Feb. 2004, p. 1. doi:10.1016/j.paerosci.2003.11.001

[17] Malik, M. R., and Balakumar, P., "Acoustic Receptivity of Mach 4.5 Boundary Layer with Leading Edge Bluntness," Theoretical and Computational Fluid Dynamics, Vol.21, No.5, Sept.2007, pp.323-342. doi:10.1007/s00162-007-0050-5

[18] Kara, K., Balakumar, P., and Kandil, O. A., "Receptivity of Hypersonic Boundary Layers Due to Acoustic Disturbances over Blunt Cone," AIAA Paper 0945, 2007.

[19] Balakumar, P., "Stability of Supersonic Boundary Layers over Blunt Wedges," AIAA Paper 3053, 2006.

[20] Shu, C. W., "Essentially Non-Oscillatory and Weighted Essentially Non-Oscillatory Schemes for Hyperbolic Conservation Laws," NASA CR-97-206253 and ICASE Report No. 97-6, 1997.

[21] Atkins, H. L., "High-Order ENO Methods for the Unsteady Compressible Navier-Stokes Equations," AIAA Paper 1557, 1991.

[22] Balakumar, P., Zhao, H., and Atkins, H., "Stability of Hypersonic Boundary Layers over a Compression Corner," AIAA Paper 2848, 2002.

[23] Kara, K., "Hypersonic Boundary Layer Receptivity to Acoustic Disturbances over Cones," Ph.D. Dissertation, Old Dominion University, 2008.

[24] Malik, M. R., and Balakumar, P., "Receptivity of Supersonic Boundary Layers to Acoustic Disturbances," AIAA Paper 5027, 2005.

A. Tumin

Associate Editor 